\title{
On the relation of protein dynamics and exciton relaxation in pigment-protein complexes: An estimation of the spectral density and a theory for the calculation of optical spectra
}

\author{
Thomas Renger and R. A. Marcus a) \\ Noyes Laboratory of Chemical Physics, Pasadena, California 91125
}

(Received 15 November 2001; accepted 25 February 2002)

\begin{abstract}
A theory for calculating time- and frequency-domain optical spectra of pigment-protein complexes is presented using a density matrix approach. Non-Markovian effects in the excitonvibrational coupling are included. A correlation function is deduced from the simulation of $1.6 \mathrm{~K}$ fluorescence line narrowing spectra of a monomer pigment-protein complex (B777), and then used to calculate fluorescence line narrowing spectra of a dimer complex (B820). A vibrational sideband of an excitonic transition is obtained, a distinct non-Markovian feature, and agrees well with experiment on B820 complexes. The theory and the above correlation function are used elsewhere to make predictions and compare with data on time-domain pump-probe spectra and frequencydomain linear absorption, circular dichroism and fluorescence spectra of Photosystem II reaction centers. (C) 2002 American Institute of Physics. [DOI: 10.1063/1.1470200]
\end{abstract}

\section{INTRODUCTION}

Understanding the complex role of proteins in charge and energy transfer reactions presents an interesting challenge for theory. In recent years the picture describing proteins as a rigid scaffold has changed. Protein dynamics is recognized as playing a significant part in the reaction. One of the more spectacular pieces of evidence on how chemical reactions can trigger conformational motion (and vice versa) comes from single molecule experiments on protein motors. ${ }^{1}$ In addition to these large scale motions on a microsecond time scale much faster reactions involving energy or charge transfer processes are influenced by small amplitude protein vibrations on a picosecond time scale. The complex structure of the proteins allows them to exhibit this extremely broad dynamic range.

The complexity of the protein is also the source of a main difficulty in modeling these systems. There is no simple unique model for the protein, and different descriptions focus on different experiments. For example, conformational motion can be neglected when interpreting subpicosecond experiments. Instead, the protein can be regarded as existing in different conformations, reflecting a static disorder. This circumstance may lead, for example, to an inhomogeneous broadening of optical transition lines of chromophores bound to the protein, or to a distribution of charge and energy transfer rates. Our aim in the present paper is to obtain dynamic variables, such as the spectral density and correlation functions, which characterize protein dynamics relevant to exciton relaxation in photosynthetic pigment-protein complexes. These functions are then incorporated into a theory of various types of optical spectra.

To obtain a realistic correlation function for the transition energies of the photosynthetic pigments, we first exam-

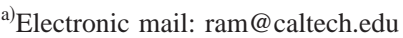

ine experiments ${ }^{2}$ on a model system consisting of an $\alpha$-helix and a bound bacteriochlorophyll molecule, the B777complex. It appears to be the only example at present of a successful step by step separation of a photosynthetic antenna complex into single-pigment-protein units, namely the preparation of the B777-complex starting from the bacterial LH1 core antenna. A structure of the B777-complex is shown in Fig. 1. It was adapted from the known structure of the bacterial $\mathrm{LH} 2$ antenna system ${ }^{3}$ by removing the $\mathrm{B} 800$ bacteriochlorophyll that is missing in the LH1 antenna complex. A low-resolution structural study exists for the LH1 complex. ${ }^{4}$ It appears that the basic pigment-protein subunits of the bacterial antennas LH1 and LH2 are related to each other and also to the bacterial reaction center. The latter is believed to be the ancestor of the plant reaction centers of photosystems I and II. ${ }^{5,6} \mathrm{~A}$ simplifying feature of the experiments ${ }^{2}$ on the B777-complex is that the specific interaction occurs between a single pigment and the protein, rather than involving, in addition, larger aggregate pigment-pigment and pigmentprotein interactions.

An exact result for linear optical absorption and fluorescence was obtained for harmonic models by Kubo and Toyozawa $^{7}$ and Lax. ${ }^{8}$ A very similar formulation was used in a harmonic model for the nonadiabatic electron transfer rate. ${ }^{9,10}$ All such approaches have in common the calculation of a transition rate between two weakly coupled electronic states which interact with a large variety of effective harmonic vibrations. Georgievskii et al. ${ }^{11}$ gave a generalization of this approach for anharmonic systems. They used linear response theory and a second-order cumulant expansion to relate the transition rate to a correlation function and a spectral density. ${ }^{11}$ The same relation is obtained in a harmonic oscillator model. Since the second-order cumulant expansion is valid for a greater class of systems, it is inferred that a description for anharmonic systems in terms of effective har- 


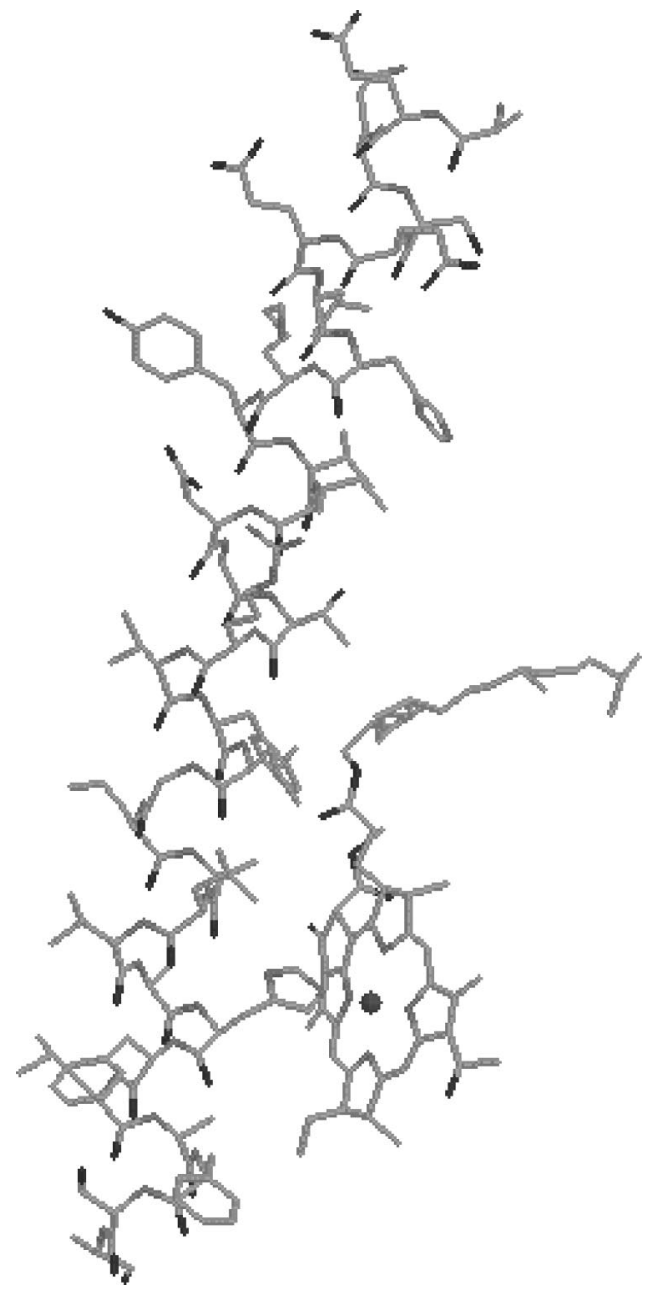

FIG. 1. Model for the B777-complex. The model has been adapted from the atomic structure of the LH2 complex (Ref. 3) (Brookhaven Protein Data file 1kzu.pdb).

monic oscillators can be found. An earlier approach with the same goal is given by Loring et al. ${ }^{12}$ and by Chandler. ${ }^{13}$

In contrast to the two-level system discussed above no exact analytic solution exists for relaxation rates of a multilevel system coupled to a large manifold of harmonic or effective harmonic oscillators. Accordingly, approximate theories have been used, as in Refs. 14-30. The multilevel Redfield theory, ${ }^{15-17}$ an example of a Markovian theory, has been applied to photosynthetic antenna systems. ${ }^{20-22}$ We recall that in a Markovian theory for a statistical density operator $\hat{\rho}(t)$ the equation of motion $d \hat{\rho}(t) / d t$ depends only on the properties of the system at $t$ and not on properties at earlier times, i.e., there are no memory effects of the earlier behavior. As noted later $\hat{\rho}(t)$ is a reduced statistical operator describing the electronic motion, reduced in the sense that it is thermally averaged over an equilibrium distribution of the vibrational motion. In general, it is the reduction in degrees of freedom from the description of the dynamics of a large system to that of a small subsystem that gives rise to nonMarkovian, or memory, effects.

If, for example, the coupling of the exciton to some selected vibrational modes is treated nonperturbatively, as was done in some effective mode models, ${ }^{31,32}$ all "memory" is automatically included for the coupling to these modes.
There is no reduced description involved. However, such an approach is computationally intensive and so has been limited to a small number of electronic pigment states and effective vibrational modes. The largest system treated so far, we believe, is a chlorophyll dimer consisting of nine electronic states coupled to two effective vibrational modes. ${ }^{32} \mathrm{An}$ alternative approach of comparable numerical intensity is given by a path integral formulation. ${ }^{33,34}$

We present an alternative and numerically less intensive approach which uses perturbation theory for part of the exciton-vibrational coupling. It will be shown how the nonMarkovian effects for a whole manifold of low-frequency protein vibrations can be included in the theory. For this purpose we determine from experiment the couplingweighted density of vibrational states of the protein, the socalled spectral density $J(\omega)$. Markovian treatments ${ }^{20-22}$ of photosynthetic antenna systems are extended in the present article by exploring non-Markovian effects for the excitonvibrational interaction. A measure of non-Markovian effects, we argue, is the production of vibrational sidebands in optical absorption and fluorescence spectra: If the interaction between the electronic and vibrational motion is such that the correlation function for the optical transition energy for the creation of the exciton decays rapidly the present process is Markovian. In such a short time there is no time for vibrations to be excited and so there are no vibrational sidebands. An earlier non-Markovian formulation of the density matrix theory ${ }^{28}$ for linear absorption and circular dichroism spectra is refined in the present article to include a more accurate summation of the exciton-vibrational coupling terms and is also extended to include the description of fluorescence line narrowing spectra and to time-domain pump-probe spectra.

When non-Markovian effects are studied the choice of an operator (the projection operator) used for the derivation of the equations of motion for the reduced statistical operator is critical. Different projection operators can be best compared by a generalized cumulant expansion method developed by Kubo ${ }^{35}$ and by van Kampen. ${ }^{36}$ As pointed out by Hashitsume et al. ${ }^{37}$ and Mukamel et al. ${ }^{38}$ the time-convolutionless projection operator technique developed by Shibata et al. $^{37,39}$ and by Tokuyama and Mori ${ }^{40}$ corresponds to a socalled partial ordering prescription (POP) in the timeordering of the cumulant expansion, ${ }^{37-39}$ whereas a more widely used projection operator technique developed by Zwanzig ${ }^{41}$ corresponds to a chronological ordering prescription (COP). ${ }^{37-39}$ The POP leads to an ordinary differential equation of motion with time-dependent coefficients for the reduced statistical operator $\hat{\rho}(t)$, whereas the COP results in an integro-differential equation for $\hat{\rho}(t)$ containing a convolution. In POP the memory effects appear in the timedependent coefficients while in COP they appear in the history of $\hat{\rho}(t)$. Besides mathematical convenience the guidelines for the choice of the time-ordering prescription may not be clear. At infinite order both prescriptions become equivalent. In the Markovian limit they also become equivalent.

One way, chosen recently by Palenberg $\mathrm{et} \mathrm{al}^{42}$ to evaluate the convergence of the two time-ordering prescriptions, is to compare with an exact result. However, the latter exists 
only for relatively simple systems. In their study of a dimer with dichotomously fluctuating site energies POP yielded the better results for the time-dependent population of dimer states. The electronic coupling between the two states was included exactly. On the other hand, for a two-level system where the levels are coupled weakly by an external field and the correlation function of the energy gap decays exponentially Mukamel showed ${ }^{43}$ how the ordering prescription is related to the stochastic properties of the energy gap: The COP equation for such a system yields the optical absorption lineshape known ${ }^{44}$ for a two-level system with a dichotomously fluctuating energy gap. The POP equation for the same system gives ${ }^{43}$ the lineshape function obtained ${ }^{44}$ for a system where the energy gap is modulated by a Gaussian random process. In the present study the choice of ordering prescription was based on the fact that the second-order POP description reduces in the one-pigment limit to the exact solution for the linear absorption because of the assumed Gaussian nature of the bath, whereas the second-order COP does not.

The paper is organized as follows: The standard spinboson type theory is used in Sec. II to extract the correlation function of the pigment transition energy from fluorescence line narrowing spectra measured ${ }^{2}$ at $1.6 \mathrm{~K}$ on B777complexes. The non-Markovian density matrix equations are discussed in Sec. III. Various phenomena in the frequencyand time-domain are treated in Sec. IV using POP and include the description of circular dichroism, linear absorption, fluorescence and pump-probe spectra. Fluorescence line narrowing spectra of B820 complexes are calculated and compared with the experimental data. ${ }^{2}$ The approximations and results are discussed in Sec. V. A summary is given in Sec. VI. Various relations used in the text are derived in Appendices A-E. The COP method and results are described in Appendix F.

The theory in the present article is applied elsewhere ${ }^{45}$ to the photophysical properties of PS-2 reaction centers of green plants, specifically to the calculation of absorption, fluorescence, circular dichroism and pump-probe spectra.

\section{CORRELATION FUNCTION OF THE ELECTRONIC ENERGY GAP: EXTRACTION FROM FLUORESCENCE LINE NARROWING SPECTRA OF B777-COMPLEXES}

The B777-complex in Fig. 1 is used here as a model system to learn about the local interaction between a pigment and the protein in photosynthetic antennae. In this section a spectral density $J(\omega)$ of the pigment-protein interaction is extracted from the recently measured ${ }^{2} 1.6 \mathrm{~K}$ fluorescence line narrowing spectra of B777-complexes measured at different excitation wavelengths. From this $J(\omega)$ the correlation function $C(t)$ of the optical transition energy of the pigment is obtained.

We use the site-selective fluorescence spectra rather than the absorption spectrum for this purpose because the static disorder in the absorption spectrum introduced by the protein and solvent environment overwhelms other details of that spectrum. In fluorescence line narrowing spectroscopy the fluorescence signal $I\left(\omega, \omega_{\text {exc }}\right)$ of selectively excited pigments depends on the excitation energy $\hbar \omega_{\text {exc }}:{ }^{46}$

$$
\begin{aligned}
& I\left(\omega, \omega_{\mathrm{exc}}\right) \\
& \quad=\int_{-\infty}^{\infty} \mathrm{d} \omega_{10} \alpha_{h}\left(\omega_{\mathrm{exc}}, \omega_{10}\right) I_{h}\left(\omega, \omega_{10}\right) P_{\mathrm{inh}}\left(\omega_{10}-\bar{\omega}_{10}\right),
\end{aligned}
$$

where $\alpha_{h}$ and $I_{h}$ are the homogeneous absorption and fluorescence spectra, respectively, of the complex with the $(0$ $\rightarrow 0)$ transition energy at $\hbar \omega_{10}$, and a Gaussian distribution function $P_{\text {inh }}\left(\omega_{10}-\bar{\omega}_{10}\right)$ is assumed, for the inhomogeneity in $0 \rightarrow 0$ transition frequencies $\omega_{10}$ of the complexes in the sample, with a maximum at $\bar{\omega}_{10}$.

To use Eq. (2.1) it is necessary to calculate the homogeneous absorption and fluorescence spectra of a pigment coupled to a variety of vibrational degrees of freedom of the system. Standard theories for treating this problem ${ }^{7,8,16}$ assume that the vibrations can be treated as effective harmonic oscillators that do not change their frequencies upon electronic excitation of the pigment. We will make the same assumption.

The calculations are based on the spin-boson type Hamiltonian: ${ }^{47}$

$$
H=H_{0}|0\rangle\left\langle 0\left|+H_{1}\right| 1\right\rangle\langle 1|,
$$

where the Hamiltonian $H_{0}$ of the electronic ground state is a Hamiltonian $H_{\text {vib }}$ of unshifted harmonic oscillators,

$$
H_{0}=H_{\mathrm{vib}}=T_{\text {nucl }}+\sum_{\xi} \frac{\hbar \omega_{\xi}}{4} Q_{\xi}^{2}
$$

and the Hamiltonian $H_{1}$ of the excited electronic state $|1\rangle$ is given as

$$
H_{1}=T_{\text {nucl }}+\hbar \omega_{10}+\sum_{\xi} \frac{\hbar \omega_{\xi}}{4}\left(Q_{\xi}+2 g_{\xi}\right)^{2},
$$

where $T_{\text {nucl }}$ denotes the kinetic energy of nuclei and $\hbar \omega_{10}$ is the energy difference between the minima of the potential energy surfaces (PES) of the excited and the ground electronic state, $|1\rangle$ and $|0\rangle$, respectively, of the pigment in the B777-complex. This Hamiltonian is the one-pigment version of the multi-pigment Hamiltonian given later. The $\omega_{\xi}$ denote the relevant frequencies and $-2 g_{\xi}$ the shift in minimum of the excited state PES relative to the ground state. For notational convenience a dimensionless coordinate $Q_{\xi}$ $=q_{\xi} \sqrt{2 \omega_{\xi} / \hbar}$ is used (e.g., Ref. 16), where the $q_{\xi}$ are the usual (mass-weighted) normal coordinates. The $Q_{\xi}$ can be written as $C_{\xi}^{+}+C_{\xi}$, i.e., in terms of the usual creation and annihilation operators of vibrational quanta of protein mode $\xi$.

The homogeneous absorption and fluorescence signals are given by ${ }^{8,16}$

$$
\begin{aligned}
& \alpha_{h}(\omega)=\frac{4 \pi^{2} \omega n}{3 \hbar c}\left|\mu_{10}\right|^{2} D_{\alpha}(\omega), \\
& I_{h}(\omega)=\frac{4 \omega^{3} n^{3}}{3 \hbar^{2} c^{3}}\left|\mu_{10}\right|^{2} D_{I}(\omega),
\end{aligned}
$$


where $\mu_{10}$ is the transition dipole moment, $n$ the refractive index of the sample and the lineshape functions $D_{\alpha / I}(\omega)$ for absorption $(\alpha)$ and fluorescence $(I) \operatorname{are}^{8,16}$

$$
D_{\alpha / I}(\omega)=\frac{1}{2 \pi} \int_{-\infty}^{\infty} \mathrm{d} t e^{ \pm \imath\left(\omega-\omega_{10}\right) t+G(t)-G(0)} .
$$

Here + refers to $\alpha$ and - to $I$. This $D_{\alpha / I}(\omega)$ contains a time-dependent function $G(t)$ of the spectral density $J(\omega),{ }^{48}$

$$
\begin{aligned}
& G(t)=\int_{0}^{\infty} d \omega\left\{(1+n(\omega)) J(\omega) e^{-i \omega t}+n(\omega) J(\omega) e^{i \omega t}\right\}, \\
& J(\omega)=\sum_{\xi} g_{\xi}^{2} \delta\left(\omega-\omega_{\xi}\right) .
\end{aligned}
$$

This $J(\omega)$ describes how the configuration of nuclei of the protein changes upon electronic excitation of the pigment. The $n(\omega)$ in Eq. (2.8) is the mean number of vibrational quanta for an oscillator of frequency $\omega$ in thermal equilibrium: ${ }^{49}$

$$
n(\omega)=\frac{1}{e^{\hbar \omega / k T}-1} .
$$

The lineshape function, Eq. (2.7), can be written as a sum of two terms: a zero-vibrational quantum $(0 \rightarrow 0)$ transition and vibrational sidebands: ${ }^{46}$

$$
\begin{aligned}
D_{\alpha / I}(\omega)= & \frac{e^{-G(0)}}{2 \pi} \int_{-\infty}^{\infty} \mathrm{d} t e^{i\left(\omega-\omega_{10}\right) t} \\
& +\frac{e^{-G(0)}}{2 \pi} \int_{-\infty}^{\infty} \mathrm{d} t e^{ \pm i\left(\omega-\omega_{10}\right) t}\left(e^{G(t)}-1\right),
\end{aligned}
$$

which can also be written as

$$
D_{\alpha / I}(\omega)=e^{-G(0)} \delta\left(\omega-\omega_{10}\right)+\phi\left( \pm\left(\omega-\omega_{10}\right)\right) .
$$

Here $\delta\left(\omega-\omega_{10}\right)$ refers to the $0 \rightarrow 0$ transition and the $\phi\left( \pm\left(\omega-\omega_{10}\right)\right)$ to the absorption (+) or fluorescence (-) vibrational sidebands.

The homogeneous lineshape functions $D_{\alpha}(\omega)$ and $D_{I}(\omega)$ are mirror symmetric with respect to the energy $\hbar \omega_{10}$, i.e., $D_{\alpha}\left(\omega-\omega_{10}\right)=D_{I}\left(\omega_{10}-\omega\right)$, reflecting the assumption that the vibrational frequencies of the effective harmonic oscillators do not depend on the electronic state of the pigment. The integrated intensity of the vibrational sideband $\int_{-\infty}^{\infty} d \omega \phi\left(\omega-\omega_{10}\right)$ relative to that of the zero-vibrational quantum transition $e^{-G(0)}$, equals $e^{G(0)}-1$, and is a measure of the exciton-vibrational coupling strength. The significance of $G(0)$ is that at temperature $T=0$, it can be shown from Eq. (2.8) to equal $S$, the well-known Huang-Rhys factor

$$
S=\int_{0}^{\infty} d \omega J(\omega)=\sum_{\xi} g_{\xi}^{2},
$$

where $g_{\xi}^{2}$ also equals the average change ${ }^{8}$ in number of vibrational quanta with energy $\hbar \omega_{\xi}$ in an optical transition from any specific initial vibrational state. Since the displacement of the minima of the potential energy surfaces of the two electronic states with respect to each other equals $-2 g_{\xi}$, the more this displacement, the larger is $S$.

The exciton-vibrational coupling is weak when $S \ll 1$, and strong when $S \gg 1$. The typical coupling in photosynthetic antenna complexes lies in an intermediate range $S$ $\approx 1,{ }^{50-53}$ a notable exception being the red-absorbing antenna states of photosystem I $(S>2) .{ }^{54-56}$

Introducing the results for the homogeneous absorption and fluorescence lineshape functions Eqs. (2.12) into Eq. (2.1), using Eqs. (2.5) and (2.6), yields the fluorescence line narrowing spectrum at a frequency $\omega$, excited at a frequency $\omega_{\text {exc }}$, which includes an inhomogeneous distribution function $P_{\text {inh }}$ having a maximum at $\omega_{10}=\bar{\omega}_{10}$,

$$
\begin{aligned}
I\left(\omega, \omega_{\mathrm{exc}}\right) \sim & \int_{0}^{\infty} \mathrm{d} \omega_{10}\left\{e^{-G(0)} \delta\left(\omega_{\mathrm{exc}}-\omega_{10}\right)\right. \\
& \left.+\phi\left(\omega_{\mathrm{exc}}-\omega_{10}\right)\right\}\left\{e^{-G(0)} \delta\left(\omega-\omega_{10}\right)\right. \\
& \left.+\phi\left(\omega_{10}-\omega\right)\right\} P_{\mathrm{inh}}\left(\omega_{10}-\bar{\omega}_{10}\right) .
\end{aligned}
$$

Equation (2.14) can be written as

$$
\begin{aligned}
I\left(\omega, \omega_{\mathrm{exc}}\right) \sim & e^{-2 G(0)} \delta\left(\omega-\omega_{\mathrm{exc}}\right) P_{\mathrm{inh}}\left(\omega_{\mathrm{exc}}-\bar{\omega}_{10}\right) \\
& +e^{-G(0)} \phi\left(\omega_{\mathrm{exc}}-\omega\right)\left(P_{\mathrm{inh}}\left(\omega_{\mathrm{exc}}-\bar{\omega}_{10}\right)\right. \\
& \left.+P_{\mathrm{inh}}\left(\omega-\bar{\omega}_{10}\right)\right)+\int \mathrm{d} \omega_{10} \phi\left(\omega_{\mathrm{exc}}-\omega_{10}\right) \\
& \times \phi\left(\omega_{10}-\omega\right) P_{\mathrm{inh}}\left(\omega_{10}-\bar{\omega}_{10}\right) .
\end{aligned}
$$

The first term on the right in Eq. (2.15) is the resonant fluorescence at the excitation wavelength and originates from the $0 \rightarrow 0$ transitions $\delta\left(\omega_{\mathrm{exc}}-\omega_{10}\right)$ in absorption followed by the $0 \rightarrow 0$ transition $\delta\left(\omega-\omega_{10}\right)$ in fluorescence. The factor $e^{-2 G(0)} P_{\text {inh }}\left(\omega_{\text {exc }}-\bar{\omega}_{10}\right)$ does not appear to have been extracted experimentally ${ }^{2}$ for the present system, because of interference by scattered light from the excitation. ${ }^{57}$

The second term on the right side of Eq. (2.15) contains two types of sidebands: The first arises from excitation of the $0 \rightarrow 0$ transition $\delta\left(\omega_{\mathrm{exc}}-\omega_{10}\right)$ followed by fluorescence yielding the sideband $\phi\left(\omega_{10}-\omega\right)$, and the second from excitation of the sideband $\phi\left(\omega_{\mathrm{exc}}-\omega_{10}\right)$ and followed by fluorescence at the $0 \rightarrow 0$ transition $\delta\left(\omega-\omega_{10}\right)$. The last term on the right hand side of Eq. (2.15) contains excitation of a sideband and fluorescence to a sideband.

The above expression simplifies when the pigmentprotein coupling is weak. We use this case as a first step in an iterative procedure to extract $J(\omega)$ from the data: For weak coupling the shape of the vibrational sideband $\phi(\omega)$ at low temperatures is the same as the shape of the spectral density $J(\omega)$, as can be seen by approximating $e^{G(t)}-1$ by $G(t)$ in Eq. (2.11) and setting $n(\omega)=0$ in Eq. (2.8). Further, since the vibrational sideband in absorption at low temperatures must be only on the high-energy side of the $0 \rightarrow 0$ transition, a $0 \rightarrow 0$ transition is the major contributor to the low-energy wing of the absorption spectrum. In this case the shape of the vibrational sideband in fluorescence line narrowing spectra is given by the $\phi\left(\omega_{\mathrm{exc}}-\omega\right)$ appearing in Eq. (2.15). The following strategy was therefore chosen to extract $J(\omega)$ from the spectrum: 


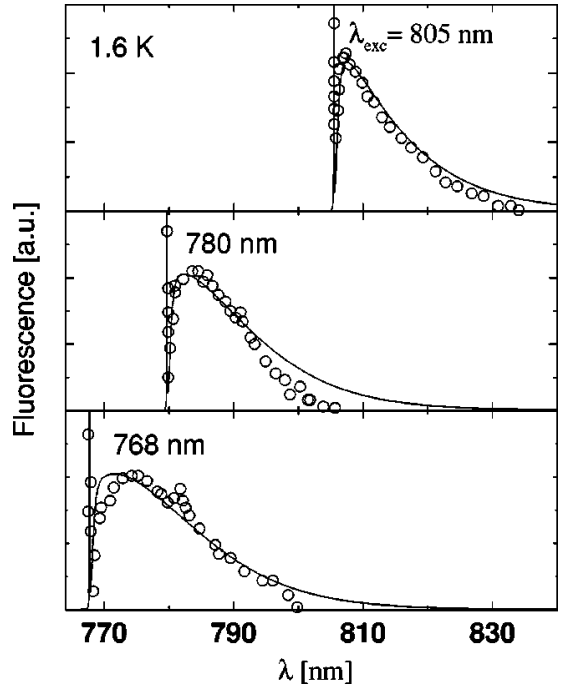

FIG. 2. Dependence of fluorescence line narrowing spectra of B777complexes on the excitation wavelength. Open circles denote the experimental values from Ref. 2 and solid lines represent the theoretical fit using the spectral density in Fig. 3.

The shape of the sideband in the fluorescence line narrowing spectrum excited in the low-energy wing of the absorption spectrum was used as a first guess for the spectral density $J(\omega)$. An empirical functional form which is an extension of earlier ${ }^{21,22,25,27,28,50}$ functional forms was used for $J(\omega)$ :

$$
J(\omega)=\sum_{i=1,2} s_{i} k_{i} \omega^{q} e^{-\left(\omega / \omega_{i}\right)^{p}} .
$$

It contains the parameters $s_{i}, \omega_{i}, p$ and $q, k_{i}$ being the normalization factors, $p /\left(\omega_{i}^{(q+1)} \Gamma((q+1) / p)\right)$, and the sum $s_{1}+s_{2}$ being equal to the $S$ of $J(\omega)$ in Eq. (2.13). An initial value $S=1$ was used, typical for pigment-protein complexes as discussed before. The fit was iterated by next calculating $\phi(\omega)$ in Eqs. (2.8)-(2.12) numerically and then calculating the spectrum from Eq. (2.15). This fit was performed for the lowest excitation energy $\hbar \omega_{\text {exc }}$ used in the experiment. Using this first estimate for the shape of $J(\omega)$, the $S=s_{1}+s_{2}$ was varied next to fit the dependence of $I\left(\omega, \omega_{\text {exc }}\right)$ on the excitation frequency $\omega_{\text {exc }}$ in Eq. (2.15). At higher excitation energies the last contribution in Eq. (2.15) involving sidebands in absorption and in emission becomes important and leads to a broadening of the sideband seen in the spectrum in Fig. 2 at short wavelengths. Since the weight of the sidebands is determined by $S$ this broadening effect can be used to estimate $S$.

The fit at the different $\omega_{\text {exc }}$ 's in Fig. 2 was obtained for $S=1.3$. The $J(\omega)$ so determined is shown in the upper half of Fig. 3. The optimized parameters are $s_{1}=0.8, s_{2}=0.5$, $\hbar \omega_{1}=0.069 \mathrm{meV}, \hbar \omega_{2}=0.24 \mathrm{meV}, p=0.5, q=3$. For $p$ $=0.5$ and $q=3$ the spectral density, Eq. (2.16), is

$$
J(\omega)=\sum_{i=1,2} \frac{s_{i}}{7 ! 2 \omega_{i}^{4}} \omega^{3} e^{-\left(\omega / \omega_{i}\right)^{1 / 2}}
$$
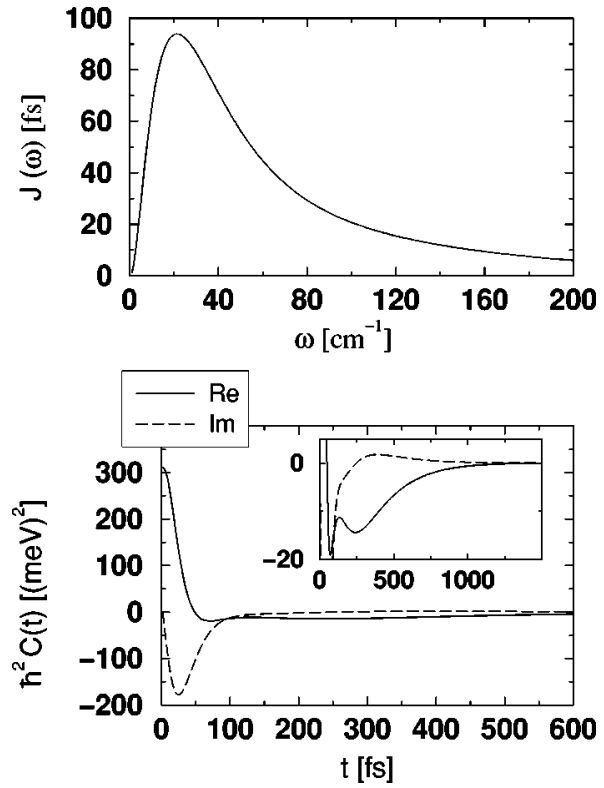

FIG. 3. Upper half: Spectral density $J(\omega)$, extracted from the fit of fluorescence line narrowing spectra of the B777-complex. Lower half: Correlation function of the pigment transition energy of B777-complexes at $T=1.6 \mathrm{~K}$. Solid and dashed lines show the real and imaginary parts, respectively. The inset in the lower half is a zoom into the small amplitude long time components of the correlation function.

The maxima of the two contributions in the above sum occur at frequencies $36 \omega_{i}$, i.e., at 20 and $70 \mathrm{~cm}^{-1}$. Because $s_{1}$ $>s_{2}$ the first maximum determines the maximum of $J(\omega)$ in Fig. 3.

If the system were treated as a continuum of harmonic vibrations, as in the Debye model of specific heat, the density of the vibrations, namely the $\Sigma_{\xi} \delta\left(\omega-\omega_{\xi}\right)$ in Eq. (2.9), would contribute a factor $\omega^{2}$ to the $J(\omega)$ and there would be a cut-off in $\omega$, because of the finite number of coordinates. The remaining dependence on $\omega$ in Eq. (2.17), $\omega \exp$ $-\left(\omega / \omega_{i}\right)^{1 / 2}$ would then arise from this cut-off and from the dependence of $g_{\xi}^{2}$ in Eq. (2.9) on $\xi$ and hence on $\omega$.

The parameters of the Gaussian distribution function $P_{\text {inh }}\left(\omega_{10}-\bar{\omega}_{10}\right)$, used in the calculation of the fluorescence line narrowing spectrum in Eq. (2.14), were determined from the linear absorption spectrum ${ }^{2}$ of the B777-complex in Fig. 4. The maximum of $P_{\text {inh }}$ occurs at a frequency $\bar{\omega}_{10}$ corresponding to a wavelength of $782 \mathrm{~nm}$ (and so shifted to the red of the absorption maximum at $777 \mathrm{~nm}$ ) and the width of the inhomogeneous distribution function (FWHM) is $600 \mathrm{~cm}^{-1} .58$

The correlation function $C(t)$ of the transition energy $X=H_{1}-H_{0}$ of the pigment is given as

$$
C(t)=\frac{1}{\hbar^{2}}\langle\delta X(t) \delta X(0)\rangle,
$$

where $\langle\cdots\rangle=\operatorname{Tr}_{\text {vib }}\left\{W_{\text {eq }} \ldots\right\}$ and denotes an average with respect to the equilibrium statistical operator of $H_{0}=H_{\mathrm{vib}}$,

$$
W_{\mathrm{eq}}=e^{-H_{\mathrm{vib}} / k T} / \operatorname{Tr}_{\mathrm{vib}}\left\{e^{-H_{\mathrm{vib}} / k T}\right\} .
$$

The $\delta X$ is the deviation of $X$ from its mean value $\langle X\rangle$, 


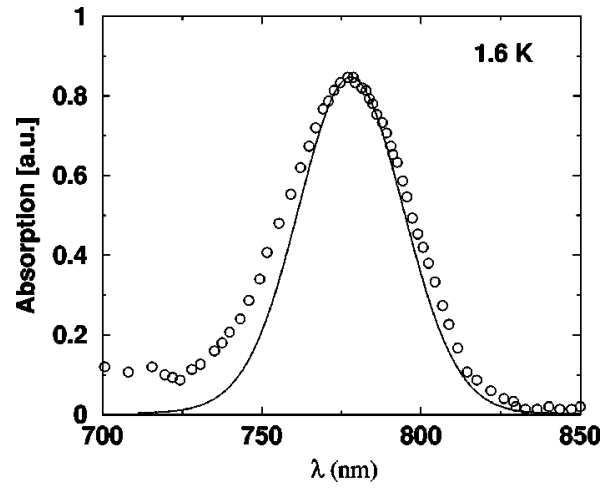

FIG. 4. Linear absorption spectrum of B777-complexes using the spectral density of Fig. 3 Open circles denote the experimental values from Ref. 2 and the solid line the calculated values.

$$
\delta X=X-\langle X\rangle=\sum_{\xi} \hbar \omega_{\xi} g_{\xi} Q_{\xi},
$$

where the time dependence is determined by $H_{\mathrm{vib}}$,

$$
\delta X(t)=e^{i H_{\mathrm{vib}} t / \hbar} \delta X e^{-i H_{\mathrm{vib}} t / \hbar} .
$$

The $C(t)$ is obtained in terms of the spectral density $J(\omega)$ (Appendix A):

$C(t)=\int_{0}^{\infty} d \omega \omega^{2}\left\{(1+n(\omega)) J(\omega) e^{-i \omega t}+n(\omega) J(\omega) e^{i \omega t}\right\}$.

As seen in the lower half of Fig. 3 the real part of $C(t)$ exhibits a strongly damped oscillation. It and the imaginary part of $C(t)$ are essentially zero after about $100 \mathrm{fs}$. About $5 \%$ of the initial correlation is seen in the inset of Fig. 3 to decay on a $500 \mathrm{fs}$ time scale. This $C(t)$ of the B777-complex will be related later to an excitonic correlation function and will be used for the description of dissipation and optical spectra in larger complexes, containing more than one pigment.

\section{DENSITY MATRIX THEORY OF EXCITON RELAXATION}

We next introduce an exciton-vibrational Hamiltonian $H$ and from it obtain the equations of motion for the excitonic density operator $\hat{\rho}(t)$. In the following $H$ is given by the sum, $H_{\text {ex }}+H_{\text {vib }}+H_{\text {ex-vib }}+H_{\text {ex-rad }}$. It contains the exciton term $H_{\mathrm{ex}}$,

$$
H_{\mathrm{ex}}=\sum_{\mu} \mathcal{E}_{\mu}|\mu\rangle\langle\mu|,
$$

where $\mu=0$ denotes the electronic ground state at energy $\mathcal{E}_{0}=0, \mu=M$ a one-exciton state at energy $\mathcal{E}_{M}, \mu=2 M$ a two-exciton state at energy $\mathcal{E}_{2 M}$, a vibrational term $H_{\text {vib }}$ in Eq. (2.3) composed of unshifted harmonic oscillators, and $H_{\text {ex-vib }}$ an exciton-vibrational coupling term,

$$
H_{\mathrm{ex}-\mathrm{vib}}=\sum_{\mu \nu} \hat{V}_{\mu \nu}|\mu\rangle\langle\nu| .
$$

The operator $\hat{V}_{\mu \nu}$ contains the coupling between one-exciton states $\mu=M, \nu=N$, and between two-exciton states $\mu$
$=2 M, \nu=2 N$, and can be expressed in terms of the eigencoefficients of the exciton states $c_{m}^{(M)}, c_{m n}^{(2 M)}$ and coupling constants $g_{\xi}^{(m)}$,

$$
\begin{aligned}
& \hat{V}_{M N}=\sum_{\xi} \sum_{m} c_{m}^{(M)} c_{m}^{(N)} \hbar \omega_{\xi} g_{\xi}^{(m)} Q_{\xi}, \\
& \hat{V}_{2 M 2 N}=\sum_{\xi} \sum_{m>n} c_{m n}^{(2 M)} c_{m n}^{(2 N)} \hbar \omega_{\xi}\left(g_{\xi}^{(m)}+g_{\xi}^{(n)}\right) Q_{\xi},
\end{aligned}
$$

all other matrix elements $\hat{V}_{\mu \nu}$ in Eq. (3.2) being zero. The c's and $\mathcal{E}_{\mu}$ are obtained ${ }^{59}$ from the one- and two-exciton state eigenvalue problems, as usual (e.g., Ref. 30). As input parameters for this diagonalization procedure the intermolecular couplings $V_{m n}$ and optical transition energies $X_{m}$ of the pigments at the minimum position of the potential energy surface of the electronic ground state are needed. ${ }^{16}$ To take into account a modulation of $X_{m}$ by the vibrations, the $X_{m}$ is expanded about the minimum position of the ground state PES. The linear dependence is described by the excitonvibrational coupling constants $g_{\xi}^{(m)}=\partial X_{m} /\left.\partial Q_{\xi}\right|_{0}$.

The Hamiltonian $H_{\text {ex-rad }}$ describing the coupling of the aggregate to the external pump and probe fields is, in the rotating wave approximation,

$$
H_{\text {ex-rad }}=\sum_{\mu \nu} F_{\mu \nu}(t)|\mu\rangle\langle\nu|,
$$

where $F_{M 0}(t)$ describes the absorptive radiative coupling of the ground state $|0\rangle$ to the one-exciton state $|M\rangle$ and $F_{2 N M}(t)$ the coupling of the one-exciton $|M\rangle$ to the twoexciton state $|2 N\rangle$,

$$
\begin{aligned}
& F_{M 0}(t)=-\sum_{s=p u, p r} E_{\Omega_{s}}(t) e^{-i \Omega_{s} t} \mu_{M}^{(s)}, \\
& F_{2 N M}(t)=-\sum_{s=p u, p r} E_{\Omega_{s}}(t) e^{-i \Omega_{s} t} \mu_{M \rightarrow 2 N}^{(s)} .
\end{aligned}
$$

The emissive radiative couplings are $F_{0 M}(t)=F_{M 0}^{*}(t)$ and $F_{M 2 N}(t)=F_{2 N M}^{*}(t)$, respectively.

The external field is characterized by the envelopes $E_{\Omega_{s}}(t)$ of the pump $(s=\mathrm{pu})$ and probe $(s=\mathrm{pr})$ pulses, their carrier frequencies $\Omega_{s}$, and their polarization vectors $\vec{e}_{s}$. The scalar products of $\vec{e}_{s}$ and the dipole moments of the excitonic transitions $\vec{\mu}_{M}$ and $\vec{\mu}_{M \rightarrow 2 N}$ are denoted by $\mu_{M}^{(s)}$ and $\mu_{M \rightarrow 2 N}^{(s)}$ :

$$
\begin{aligned}
& \mu_{M}^{(s)}=\vec{e}_{s} \cdot \vec{\mu}_{M} \equiv \vec{e}_{s} \cdot \sum_{m} c_{m}^{(M)} \vec{\mu}_{m}, \\
& \mu_{M \rightarrow 2 N}^{(s)}=\vec{e}_{s} \cdot \vec{\mu}_{M \rightarrow 2 N} \equiv \vec{e}_{s} \cdot \sum_{k>l} c_{k l}^{(2 N)}\left(c_{l}^{(M)} \vec{\mu}_{k}+c_{k}^{(M)} \vec{\mu}_{l}\right),
\end{aligned}
$$

where the local dipole moment $\vec{\mu}_{m}$ characterizes the $S_{0}$ $\rightarrow S_{1}$ transition of the pigment at site $m$, and $\vec{\mu}_{M} \equiv \vec{\mu}_{0 \rightarrow M}$ is the dipole moment of the excitation of the one-exciton state $|M\rangle$ and $\vec{\mu}_{M \rightarrow 2 N}$ is the dipole moment of the optical transition from the one-exciton state $|M\rangle$ to the two-exciton state $|2 N\rangle$. 
In treating the dynamics of the excitons a reduced statistical operator $\hat{\rho}(t)$ is introduced, ${ }^{15,16}$ as noted earlier, $\hat{\rho}(t)$ $=\operatorname{tr}_{\mathrm{vib}}\{\hat{W}(t)\}$, where the statistical operator $\hat{W}(t)$ of the entire system is reduced by taking the trace with respect to all vibrational coordinates of the protein and the pigments. When there is no coupling to external light fields the system is in its ground state and the statistical operator $\hat{W}(t)$ factorizes into a vibrational and an excitonic part $\widetilde{W}(t)$ $=W_{\text {eq }}|0\rangle\langle 0|$. This factorization occurs prior to a time $t_{0}$, the time of the first interaction with an external field. This $W_{\mathrm{eq}}$ is the equilibrium statistical operator of the vibrations in Eq. (2.19), with $H_{\text {vib }}$ given by Eq. (2.3).

The coupling with the external field then causes the excitonic and vibrational part to depart from equilibrium. Because the excited states are coupled to the vibrations the statistical operator of the optically excited states does not factorize as for the ground state. The density matrix theory provides a way to treat the correlations between excitonic and vibrational degrees of freedom. ${ }^{60}$

Different techniques can be used to obtain an equation of motion for the reduced statistical operator $\hat{\rho}(t)$. The following equations (3.9)-(3.12) apply to the present nonMarkovian treatment of the exciton-vibrational problem. A second-order cumulant expansion ${ }^{35}$ for the time-evolution operator yields ${ }^{38}$

$$
\frac{\partial}{\partial t} \hat{\rho}(t)=-i\left(\mathcal{L}_{\text {ex }}+\mathcal{L}_{\text {ex -rad }}(t)\right) \hat{\rho}(t)+\left.\frac{\partial}{\partial t} \hat{\rho}(t)\right|_{\text {dissi }},
$$

where the Liouville operator $\mathcal{L}_{\text {ex }}$,

$$
\mathcal{L}_{\mathrm{ex}} \hat{\rho}(t)=\frac{1}{\hbar}\left[H_{\mathrm{ex}}, \hat{\rho}(t)\right]
$$

describes the dissipation-free dynamics of the excitonic system, and the $\mathcal{L}_{\text {ex-rad }}(t)$ the exciton-radiational coupling,

$$
\mathcal{L}_{\text {ex-rad }}(t) \hat{\rho}(t)=\frac{1}{\hbar}\left[H_{\text {ex-rad }}(t), \hat{\rho}(t)\right]
$$

the square brackets denoting commutators, as usual. The last term in Eq. (3.9) contains the dissipative part due to the exciton-vibrational coupling. Different prescription schemes for the cumulant expansion have been used to obtain this dissipative part, ${ }^{35,38}$ as discussed in the Introduction. We use the partial ordering prescription (POP) $)^{38,61}$ first. As discussed later it is the more appropriate of the two for the present problem. Results obtained from the alternative chronological ordering prescription $(\mathrm{COP})^{26,28,38,41}$ are given later for comparison.

The dissipative part of the equation of motion obtained from second-order POP is ${ }^{38}$

$$
\begin{aligned}
\left.\frac{\partial}{\partial t} \hat{\rho}(t)\right|_{\mathrm{dissi}}= & -\frac{1}{\hbar^{2}} \int_{0}^{t-t_{0}} d \tau\left\langle\mathcal{L}_{\mathrm{ex}-\mathrm{vib}}(t) e^{-i \mathcal{L}_{\mathrm{ex}} \tau}\right. \\
& \left.\times \mathcal{L}_{\mathrm{ex}-\mathrm{vib}}(t-\tau) e^{i \mathcal{L}_{\mathrm{ex}} \tau}\right\rangle \hat{\rho}(t),
\end{aligned}
$$

where the \langle\rangle in Eq. (3.12) denotes an equilibrium average over the vibrational degrees of freedom $\langle\hat{O}\rangle=\operatorname{Tr}_{\text {vib }}\left\{\hat{O} W_{\text {eq }}\right\}$, $W_{\text {eq }}$ being the equilibrium statistical operator of the vibrations given in Eq. (2.19). The time $t_{0}$ in Eq. (3.12) is a time for onset of an external field. For finite pulse widths the choice of $t_{0}$ is somewhat arbitrary. One has to cut off the pulse for earlier times, which is an approximation that is not necessary in the COP treated in Appendix F. Any dependence of dissipation on the intensity of the external field has been neglected in Eq. (3.12). The Liouville operator $\mathcal{L}_{\text {ex-vib }}(t)$ in Eq. (3.12) acts on an arbitrary operator $\hat{O}$ according to $\mathcal{L}_{\text {ex-vib }}(t) \hat{O}=1 / \hbar\left[H_{\text {ex-vib }}(t), \hat{O}\right]$, with $H_{\text {ex-vib }}(t)$ being the exciton-vibrational Hamiltonian in the interaction representation,

$$
H_{\mathrm{ex}-\mathrm{vib}}(t)=e^{i H_{\mathrm{vib}} t / \hbar} H_{\mathrm{ex}-\mathrm{vib}} e^{-i H_{\mathrm{vib}} t / \hbar} .
$$

The operator $e^{i \mathcal{L}_{\mathrm{ex}} \tau}$ in Eq. (3.12) acts on an arbitrary operator $\hat{\mathrm{O}}$ as $e^{i \mathcal{L}_{\mathrm{ex}} \tau} \hat{O}=e^{i H_{\mathrm{ex}} \tau / \hbar} \hat{O} e^{-i H_{\mathrm{ex}} \tau / \hbar}$. The reduced statistical operator $\hat{\rho}(t)$ in Eqs. (3.9)-(3.12) is then expanded in terms of exciton states $\mu, \nu$,

$$
\hat{\rho}(t)=\sum_{\mu \nu} \rho_{\mu \nu}(t)|\mu\rangle\langle\nu| .
$$

The equations of motion for the exciton density matrix $\rho_{\mu \nu}(t)$ are given in Appendix B in terms of the correlation function $C(t)$ of the optical energy gap of the pigments. The various phenomena are treated in the following sections using these equations.

\section{TREATMENT OF PHENOMENA}

\section{A. Linear absorption, circular dichroism and fluorescence \\ 1. Linear absorption}

The linear absorption spectrum $\alpha(\omega)$ is obtained as the real part of a Fourier-Laplace transform of the dipoledipole correlation function $D(t),{ }^{43,62} \alpha(\omega) \propto \operatorname{Re} \int_{0}^{\infty} d t e^{i \omega t} D(t)$, where $D(t)=\Sigma_{M}\left|\mu_{M}\right|^{2} \rho_{M 0}(t)$ and $\rho_{M 0}(0)=1$. The absorption spectrum then is

$$
\alpha(\omega) \propto \sum_{M}\left|\mu_{M}\right|^{2} D_{M}(\omega),
$$

with the lineshape function $D_{M}(\omega)$,

$$
D_{M}(\omega)=\operatorname{Re} \int_{0}^{\infty} d t e^{i \omega t} \rho_{M 0}(t) .
$$

Using the quantum master equation in Appendix B the equation of motion for $\rho_{M 0}(t)$ is

$$
\begin{aligned}
\frac{\partial}{\partial t} \rho_{M 0}(t)= & -i \omega_{M 0} \rho_{M 0}(t) \\
& -\sum_{K, L} \int_{0}^{t} d \tau C_{M K K L}(\tau) e^{i \omega_{L K} \tau} \rho_{L 0}(t),
\end{aligned}
$$

where $\hbar \omega_{L K}=\mathcal{E}_{L}-\mathcal{E}_{K}$ and $\hbar \omega_{M 0}=\mathcal{E}_{M}$ these $\mathcal{E}_{M}$ being the exciton energies in Eq. (3.1). The correlation function $C_{M K K L}(\tau)$ in Eq. (4.3) is

$$
C_{M N K L}(t)=\sum_{m n} c_{m}^{(M)} c_{m}^{(N)} c_{n}^{(K)} c_{n}^{(L)}\left\langle\delta X_{m}(t) \delta X_{n}(0)\right\rangle .
$$

The latter contains the eigencoefficients of exciton states $c_{m}^{(M)}$ and a two-site correlation function,

$$
\left\langle\delta X_{m}(t) \delta X_{n}(0)\right\rangle=\left\langle e^{i H_{\mathrm{vib}} t / \hbar} \delta X_{m} e^{-i H_{\mathrm{vib}} t / \hbar} \delta X_{n}\right\rangle .
$$


The time-dependence in Eq. (4.5) is the same as that in Eqs. (2.21) and (3.13). The $\delta X_{m}$ is the deviation of the transition energy of the $m$ th pigment from its mean value,

$$
\delta X_{m}=X_{m}-\left\langle X_{m}\right\rangle=\sum_{\xi} \hbar \omega_{\xi} g_{\xi}^{(m)} Q_{\xi}
$$

For $m \neq n$, Eq. (4.5) describes the correlation of the modulation of electronic energies at site (i.e., pigment) $m$ with that at site $n$.

We next introduce ${ }^{22}$ a radius $R_{c}$ for the correlations of protein vibrations at different sites, assuming an exponential decay for the dependence on the distance $R_{m n}$ between the pigments $m$ and $n$ :

$$
\left\langle\delta X_{m}(t) \delta X_{n}(0)\right\rangle=e^{-R_{m n} / R_{c}}\langle\delta X(t) \delta X(0)\rangle .
$$

The $\left\langle\delta X_{m}(t) \delta X_{m}(0)\right\rangle$ has $R_{m n}=0$ and will be assumed to be site-independent and is given by the $C(t)$ in Eq. (2.22). The $C(t)$ appears in the lower half of Fig. 3 and, we recall, was calculated from the spectral density $J(\omega)$ extracted from fluorescence line narrowing spectra of the B777-complex.

Using Eqs. (2.22) and (4.7) the correlation function in Eq. (4.4) can be written as

$$
C_{M N K L}(t)=\gamma_{M N K L} C(t),
$$

with

$$
\gamma_{M N K L}=\sum_{m, n} e^{-R_{m n} / R_{c}} c_{m}^{(M)} c_{m}^{(N)} c_{n}^{(K)} c_{n}^{(L)}
$$

The secular approximation, ${ }^{15,16,62}$ which neglects certain oscillating parts in the equation of motion by setting $L=M$ in Eq. (4.3), is used next. It is discussed in Appendix B and simplifies Eq. (4.3) to yield a homogeneous equation that can be solved analytically:

$\frac{\partial}{\partial t} \rho_{M 0}(t)=\left(-i \omega_{M 0}-\sum_{K} \gamma_{M K} \int_{0}^{t} d \tau C(\tau) e^{i \omega_{M K} \tau}\right) \rho_{M 0}(t)$,

$\gamma_{M K} \equiv \gamma_{M K K M}$

The solution of Eq. (4.10) is

$\rho_{M 0}(t)=\exp \left\{-i \omega_{M 0} t-\sum_{K} \gamma_{M K} \int_{0}^{t} d \tau(t-\tau) C(\tau) e^{i \omega_{M K} \tau}\right\}$.

For simplicity, a Markov approximation will next be applied to the off-diagonal part of the exciton-vibrational coupling, namely to the terms in Eq. (4.12) with $K \neq M$. This approximation is valid either when the $K=M$ terms dominate the exciton-vibrational coupling $\left(\gamma_{M M} \gg\left|\gamma_{M K}\right|\right)$ or, when on a coarse grained time axis, $t \gg 1 /\left|\omega_{M K}\right|$. The latter condition follows from the oscillating factor $e^{i \omega_{M K} \tau}$ in Eq. (4.12) which tends to cancel the contributions to the integral for times $\tau$ $\gg 1 /\left|\omega_{M K}\right|$. The diagonal terms with $M=K$ do not contain such an oscillating factor. In the presence of static disorder the respective coupling constants $\gamma_{M M}$ are larger than the $\left|\gamma_{M K}\right|$ appearing in the off-diagonal terms, as discussed later.
In this way an exact treatment in Eq. (4.12) is used, however, for the diagonal part $\gamma_{M M}$ of the exciton-vibrational coupling.

The integral in Eq. (4.12) for the off-diagonal terms $K$ $\neq M$ then becomes

$$
\begin{aligned}
& \gamma_{M K} \int_{0}^{t} d \tau(t-\tau) C(\tau) e^{i \omega_{M K} \tau} \\
& \quad \approx \gamma_{M K} t \int_{0}^{\infty} d \tau C(\tau) e^{i \omega_{M K} \tau}=\gamma_{M K} t \widetilde{C}\left(\omega_{M K}\right) .
\end{aligned}
$$

The non-Markovian solution of Eq. (4.12) is given for comparison in Appendix C. The Fourier-Laplace transform $\widetilde{C}\left(\omega_{M K}\right)$ of the correlation function $C(\tau)$ is related to the local spectral density $J(\omega)$ (Appendix A):

$$
\widetilde{C}\left(\omega_{M K}\right)=\widetilde{C}^{(\operatorname{Re})}\left(\omega_{M K}\right)+i \widetilde{C}^{(\operatorname{Im})}\left(\omega_{M K}\right),
$$

by

$$
\widetilde{C}^{(\operatorname{Re})}(\omega)=\pi \omega^{2}\{(1+n(\omega)) J(\omega)+n(-\omega) J(-\omega)\},
$$

with $\omega=\omega_{M K}$. We note that in Eq. (4.15), $J(\omega)=0$ for $\omega$ $<0,{ }^{63}$ as seen from Eq. (2.9). The $\widetilde{C}^{(\operatorname{Im})}\left(\omega_{M K}\right)$ satisfies a Kramers-Kronig relation, ${ }^{64}$

$$
\widetilde{C}^{(\operatorname{Im})}\left(\omega_{M K}\right)=\frac{1}{\pi} \wp \int_{-\infty}^{\infty} d \omega \frac{\widetilde{C}^{(\operatorname{Re})}(\omega)}{\omega_{M K}-\omega} .
$$

The diagonal part of the exciton-vibrational coupling is taken into account exactly in the integral $\gamma_{M M} \int_{0}^{t} d \tau(t$ $-\tau) C(\tau)=\gamma_{M M}\left(-i\left(E_{\lambda} / \hbar\right) t-G(t)+G(0)\right)$, where the function $G(t)$ was introduced in Eq. (2.8) and where

$$
E_{\lambda}=\int_{0}^{\infty} d \omega \hbar \omega J(\omega)
$$

is the reorganization energy. $E_{\lambda}$ is calculated for the present $J(\omega)$ to be $102 \mathrm{~cm}^{-1}$.

The lineshape function for absorption is next obtained from

$$
D_{M}(\omega)=\operatorname{Re} \int_{0}^{\infty} d t e^{i\left(\omega-\widetilde{\omega}_{M 0}\right) t} e^{G_{M}(t)-G_{M}(0)} e^{-t / \tau_{M}},
$$

where the $0 \rightarrow 0$ transition from the ground state $|0\rangle$ to an exciton state $|M\rangle$ occurs at $\widetilde{\omega}_{M 0}$,

$$
\widetilde{\omega}_{M 0}=\omega_{M 0}-\gamma_{M M} E_{\lambda} / \hbar+\sum_{K \neq M} \gamma_{M K} \widetilde{C}^{(\operatorname{Im})}\left(\omega_{M K}\right) .
$$

The time-dependent function $G_{M}(t)$ in Eq. (4.18) is

$$
G_{M}(t)=\gamma_{M M} G(t),
$$

and the inverse dephasing time

$$
\tau_{M}^{-1}=\frac{1}{2} \sum_{K} k_{M \rightarrow K}
$$

is determined by the rate constants $k_{M \rightarrow K}$ of relaxation from exciton state $|M\rangle$ to exciton state $|K\rangle$,

$$
k_{M \rightarrow K}=2 \gamma_{M K} \widetilde{C}^{(\operatorname{Re})}\left(\omega_{M K}\right) .
$$




\section{Circular dichroism}

For the calculation of the CD spectrum the dipole strength $\left|\mu_{M}\right|^{2}$ in Eq. (4.1) is replaced by the rotational strength $r_{M}$ of the excitonic transition,

$$
C D(\omega) \propto \sum_{M} r_{M} D_{M}(\omega),
$$

where

$$
r_{M}=\sum_{m>n} c_{m}^{(M)} c_{n}^{(M)} \vec{R}_{m n} \cdot \vec{\mu}_{m} \times \vec{\mu}_{n} .
$$

Here $\vec{R}_{m n}$ is a vector from site $m$ to site $n$ and the $\cdot$ and $\times$ denote the usual scalar and vector products.

\section{Fluorescence}

The density matrix theory used thus far considers equilibrium fluctuations about the equilibrium position of nuclei of the electronic ground state. When the assumption is made that a rapid vibrational relaxation occurs in the excitonic states it is useful in treating the fluorescence from state $|M\rangle$ to introduce a coordinate transformation of the Hamiltonian to coordinates relative to the equilibrium position of the $M$ th excitonic PES (Appendix D). The shifted electronic energies $\hbar \omega_{K 0}^{\prime}$ are given by Eq. (4.25), including $K=M$ and $K \neq M$,

$$
\hbar \omega_{K 0}^{\prime}=\hbar \omega_{K 0}-2 E_{\lambda} \gamma_{M M K K},
$$

with $\gamma_{M M K K}$ following from Eq. (4.9):

$$
\gamma_{M M K K}=\sum_{m, n}\left(c_{m}^{(M)}\right)^{2}\left(c_{n}^{(K)}\right)^{2} e^{-R_{m n} / R_{c}} .
$$

One has thus obtained a modified exciton-vibrational coupling Hamiltonian resulting from the shifted equilibrium position. Using this transformed Hamiltonian and the resulting exciton-vibrational correlation functions, the quantum master equation (4.10) is used in Appendix E to obtain an expression for the fluorescence signal,

$$
I(\omega) \propto \sum_{M} \frac{e^{-\hbar \omega_{M 0} / k T}}{\sum_{N} e^{-\hbar \omega_{N 0} / k T}}\left|\mu_{M}\right|^{2} D_{M}^{\prime}(\omega),
$$

in terms of the lineshape function, $D_{M}^{\prime}(\omega)$,

$$
D_{M}^{\prime}(\omega)=\operatorname{Re} \int_{0}^{\infty} d t e^{i\left(\omega-\widetilde{\omega}_{M 0}^{\prime}\right) t} e^{\left(G_{M}(t)\right)^{*}-G_{M}(0)} e^{-t / \tau_{M}^{\prime}},
$$

where the asterisk denotes the complex conjugate and where

$$
\widetilde{\omega}_{M 0}^{\prime}=\omega_{M 0}-\gamma_{M M} E_{\lambda} / \hbar+\sum_{K \neq M} \gamma_{M K} \widetilde{C}^{(\operatorname{Im})}\left(\omega_{M K}^{\prime}\right)
$$

and

$$
1 / \tau_{M}^{\prime}=\sum_{K} \gamma_{M K} \widetilde{C}^{(\mathrm{Re})}\left(\omega_{M K}^{\prime}\right) .
$$

Here, $\omega_{M K}^{\prime}=\omega_{M 0}^{\prime}-\omega_{K 0}^{\prime}$ and the fact that $\widetilde{C}^{(\mathrm{Re})}(0)=0$ was used.

The lineshape functions for absorption and circular dichroism $D_{M}(\omega)$ in Eq. (4.18) and the $D_{M}^{\prime}(\omega)$ for fluorescence in Eq. (4.28) are used in a later section to calculate fluorescence line narrowing spectra of B820 complexes. These equations were also used in our recent application ${ }^{45}$ to calculate various optical spectra of Photosystem II reaction centers.

\section{B. Time-resolved pump-probe spectra}

A now standard theory ${ }^{14}$ is next used in conjunction with the present non-Markovian density matrix description to calculate pump-probe spectra. We first recall the relevant expression. ${ }^{14}$ In a pump-probe experiment the absorption of a weak probe pulse is measured as a function of the delay time $\tau_{d}$ after the pump pulse. The pump-probe signal $\Delta \alpha\left(\tau_{d}\right)$ is defined as the difference between the absorption $\alpha\left(\tau_{d}\right)$, measured by the probe pulse delayed for $\tau_{d}$ with respect to a pump pulse, and the absorption $\alpha_{0}$ measured by the probe pulse alone. The energy loss $S(t)$ of the probe pulse in the sample can be calculated from the light-induced polarization $P(t)$ according to ${ }^{14}$

$$
S(t)=2 \Omega_{\mathrm{pr}} E_{\Omega_{\mathrm{pr}}}(t) \operatorname{Im}\left\{e^{i \Omega_{\mathrm{pr}} t} P(t)\right\},
$$

where $P(t)$ contains terms of first- and third-order in the external field. Fifth- and higher-order terms are neglected. The nonlinear polarization $P(t)$ is

$$
\begin{aligned}
P(t)= & n_{\mathrm{agg}}\left(\sum_{M} \mu_{M}^{(\mathrm{pr})}\left(\rho_{M 0}^{(1)}(t)+\rho_{M 0}^{(3)}(t)\right)\right. \\
& \left.+\sum_{M, 2 N} \mu_{M \rightarrow 2 N}^{(\mathrm{pr})}\left(\rho_{2 N M}^{(1)}(t)+\rho_{2 N M}^{(3)}(t)\right)\right),
\end{aligned}
$$

where $n_{\text {agg }}$ is the number density of aggregates in the sample. The detector in a pump-probe experiment measures a timeintegrated signal,

$$
\alpha \propto \int_{0}^{\infty} d t S(t) .
$$

Only the third-order terms in Eq. (4.32) survive the difference $\Delta \alpha(\tau)$, and the pump-probe signal can be written as ${ }^{14}$

$$
\Delta \alpha\left(\tau_{d}\right)=\int_{-\infty}^{+\infty} d \omega \Delta \alpha_{\mathrm{disp}}\left(\tau_{d}, \omega\right),
$$

with the dispersed signal being

$$
\begin{aligned}
\Delta \alpha_{\text {disp }}\left(\tau_{d}, \omega\right) \propto & \frac{\Omega_{\mathrm{pr}}}{\pi} \operatorname{Im}\left\{\widetilde { E } _ { \Omega _ { \mathrm { pr } } } ( \Omega _ { \mathrm { pr } } - \omega ) \left(\sum_{M} \mu_{M}^{(\mathrm{pr})} \widetilde{\rho}_{M 0}^{(3)}(\omega)\right.\right. \\
& \left.\left.+\sum_{M, 2 N} \mu_{M \rightarrow 2 N}^{(\mathrm{pr})} \widetilde{\rho}_{2 N M}^{(3)}(\omega)\right)\right\}
\end{aligned}
$$

with the scalar products $\mu_{M}^{(\mathrm{pr})}$ and $\mu_{M \rightarrow 2 N}^{(\mathrm{pr})}$ defined in Eq. (3.8). Instead of observing a time-integrated signal $\Delta \alpha\left(\tau_{d}\right)$ by a single detector the probe pulse signal can be dispersed in a monochromator and its frequency dependence measured, the dispersed signal being the $\Delta \alpha_{\text {disp }}\left(\tau_{d}, \omega\right)$ in Eqs. (4.34) and (4.35). The polarization terms $\widetilde{\rho}_{M 0}^{(3)}(\omega)$ and $\widetilde{\rho}_{2 N M}^{(3)}(\omega)$ in Eq. (4.35) are obtained from a hierarchy of equations for the density matrix $\rho_{\mu \nu}^{(n)}(t)$ and a Fourier transformation. Here, $\rho_{\mu \nu}^{(n)}(t)$ is of $n$ th-order in the external fields in the expansion 


$$
\rho_{\mu \nu}(t)=\sum_{n} \rho_{\mu \nu}^{(n)}(t)
$$

The different orders $n$ in $(\partial / \partial t) \rho_{\mu \nu}^{(n)}=-i \omega_{\mu \nu} \rho_{\mu \nu}^{(n)}$ $+\left.(\partial / \partial t) \rho_{\mu \nu}^{(n)}\right|_{\text {dissi }}+\left.(\partial / \partial t) \rho_{\mu \nu}^{(n)}\right|_{\text {ex-rad }}$ are generated by the field part of the equations of motion,

$$
\left.\frac{\partial}{\partial t} \rho_{\mu \nu}^{(n)}\right|_{\text {ex-rad }}=\frac{1}{i \hbar} \sum_{\kappa}\left(F_{\mu \kappa}(t) \rho_{\kappa \nu}^{(n-1)}(t)-F_{\kappa \nu}(t) \rho_{\mu \kappa}^{(n-1)}(t)\right) .
$$

The hierarchy starts with the zeroth-order density matrix element, taken as $\rho_{\mu \nu}^{(0)}=\delta_{\mu 0} \delta_{\nu 0}$.

It is assumed that the pump pulse acts before the probe pulse and that the vibrational relaxation on the PES of the exciton is fast enough that the probe pulse detects vibrationally equilibrated exciton populations and that any coherences between different exciton states have decayed. In this case the second-order density matrix elements reduce to $\rho_{\mu \nu}^{(2)}(t) \approx \delta_{\mu \nu} \delta_{\mu M} \rho_{M M}^{(2)}(t)$.

The equations of motion for the diagonal elements, the exciton state occupation probabilities $\rho_{M M}^{(2)}(t)$, are given by Eq. (4.38) using a Markov approximation for the offdiagonal exciton-vibrational coupling as before,

$$
\begin{aligned}
\frac{\partial}{\partial t} \rho_{M M}^{(2)}(t)= & -\frac{2}{\tau_{M}} \rho_{M M}^{(2)}(t)+\Sigma_{K} k_{K \rightarrow M} \rho_{K K}^{(2)}(t) \\
& +\frac{2}{\hbar} E_{\Omega_{\mathrm{pu}}} \mu_{M}^{(\mathrm{pu})} \operatorname{Im}\left\{e^{i \Omega_{\mathrm{pu}} t} \rho_{M 0}^{(1)}(t)\right\},
\end{aligned}
$$

where the dephasing constants $\tau_{M}^{-1}$ and rate constants $k_{M \rightarrow K}$ are given by Eqs. (4.21)-(4.22).

The first-order density matrix element $\rho_{M 0}^{(1)}(t)$ on the right-hand side of Eq. (4.38) is obtained as (Appendix B)

$$
\begin{aligned}
\rho_{M 0}^{(1)}(t)= & \frac{i \mu_{M}^{(\mathrm{pu})}}{2 \pi \hbar} e^{G_{M}(t)} \int_{-\infty}^{+\infty} d \omega \frac{e^{-i \omega t}}{\tau_{M}^{-1}-i\left(\omega-\widetilde{\omega}_{M 0}\right)} \\
& \times \int_{0}^{+\infty} d \tau e^{i\left(\omega-\Omega_{\mathrm{pu}}\right) \tau} e^{-G_{M}(\tau)} E_{\Omega_{\mathrm{pu}}}(\tau) .
\end{aligned}
$$

A delta function-shaped probe pulse $A_{\mathrm{pr}} \vec{e}_{\mathrm{pr}} \delta\left(t-t_{\mathrm{pr}}\right)$ at time $t_{\mathrm{pr}}=t_{\mathrm{pu}}+\tau_{d}$ is assumed and leads to the third-order density matrix elements, arising from the field parts of the equations of motion for $\rho_{\mu \nu}^{(3)}(t)$,

$$
\begin{aligned}
\rho_{M 0}^{(3)}(t) & =\rho_{M 0}^{(3)}\left(t, \rho_{00}^{(2)}\right)+\rho_{M 0}^{(3)}\left(t, \rho_{M M}^{(2)}\right) \\
& \equiv \rho_{M 0}^{(3)}(t, 0)+\rho_{M 0}^{(3)}(t, M) .
\end{aligned}
$$

In this and subsequent equations we distinguish between terms arising from the ground state population $\rho_{00}^{(2)}(t)$ and those arising from the excited state population $\rho_{M M}^{(2)}(t)$.

In the limit of fast vibrational relaxation considered, as discussed in Appendix D, the statistical operator of the exciton state $\mu$ at time $t_{\mathrm{pr}}$ is $W_{\text {eq }}(M) \rho_{M M}^{(2)}\left(t_{\mathrm{pr}}\right)$. Here, $W_{\mathrm{eq}}(M)$ denotes the equilibrium statistical operator for the vibrations in the $M$ th PES. The equations of motion for the third-order density matrix elements are then obtained as

$$
\begin{aligned}
\frac{\partial}{\partial t} \rho_{M 0}^{(3)}(t, 0)= & -i\left(\omega_{M 0}+\Sigma_{K} \gamma_{M K} \int_{0}^{t-t_{\mathrm{pr}}} d \tau C(\tau) e^{i \omega_{M K} \tau}\right) \\
& \times \rho_{M 0}^{(3)}(t, 0)-\frac{i}{\hbar} \mu_{M}^{(\mathrm{pr})} A_{\mathrm{pr}} \delta\left(t-t_{\mathrm{pr}}\right) \rho_{00}^{(2)}(t) \\
\frac{\partial}{\partial t} \rho_{M 0}^{(3)}(t, M)= & -i\left(\omega_{M 0}^{\prime}+\Sigma_{K} \gamma_{M K}^{\prime} \int_{0}^{t-t_{\mathrm{pr}}} d \tau C(\tau) e^{i \omega_{M K}^{\prime} \tau}\right) \\
& \times \rho_{M 0}^{(3)}(t, M)-\frac{i}{\hbar} \mu_{M}^{(\mathrm{pr})} A_{\mathrm{pr}} \delta\left(t-t_{\mathrm{pr}}\right) \rho_{M M}^{(2)}(t)
\end{aligned}
$$

and

$$
\begin{aligned}
\frac{\partial}{\partial t} \rho_{2 N M}^{(3)}(t)= & -i \omega_{2 N M}^{\prime} \rho_{2 N M}^{(3)}(t) \\
& -\left(\sum_{2 K} \gamma_{2 N 2 K}^{\prime} \int_{0}^{t-t_{\mathrm{pr}}} d \tau C(\tau) e^{i \omega_{2 N 2 K}^{\prime} \tau}\right. \\
& \left.+\sum_{K} \gamma_{M K}^{\prime} \int_{0}^{t-t_{\mathrm{pr}}} d \tau C^{*}(\tau) e^{i \omega_{K M}^{\prime} \tau}\right) \rho_{2 N M}^{(3)}(t) \\
& -\frac{i}{\hbar} \mu_{M \rightarrow 2 N}^{(\mathrm{pr})} A_{\mathrm{pr}} \delta\left(t-t_{\mathrm{pr}}\right) \rho_{M M}^{(2)}(t)
\end{aligned}
$$

The Fourier transforms of Eqs. (4.41)-(4.43), and the Fourier transform of the probe pulse enter into Eq. (4.35) for the dispersed pump-probe signal. Three contributions are then obtained from Eq. (4.35): ground state bleaching $\mathrm{GB}(\omega)$, stimulated emission $\operatorname{SE}\left(\omega, \tau_{d}\right)$, and excited state absorption $\operatorname{ESA}\left(\omega, \tau_{d}\right)$,

$$
\Delta \alpha_{\mathrm{disp}}\left(\omega, \tau_{d}\right) \propto \mathrm{GB}(\omega)+\mathrm{SE}\left(\omega, \tau_{d}\right)+\operatorname{ESA}\left(\omega, \tau_{d}\right) .
$$

The $\mathrm{GB}(\omega)$ is ${ }^{105}$

$$
\mathrm{GB}(\omega)=-\sum_{M}\left(\mu_{M}^{(\mathrm{pr})}\right)^{2} D_{M}(\omega) \sum_{K} P_{K}\left(t_{\mathrm{pr}}\right),
$$

where the equation $\rho_{00}^{(2)}(t)=-\Sigma_{K} \rho_{K K}^{(2)}(t)$, resulting from the identity $\Sigma_{\mu} \rho_{\mu \mu}=1$, was used,

$$
P_{K}(t)=\rho_{K K}^{(2)}(t),
$$

and $D_{M}(\omega)$ is the lineshape function of absorption, Eq. (4.18). The $\operatorname{SE}\left(\omega, \tau_{d}\right)$ is described by ${ }^{105}$

$$
\operatorname{SE}\left(\omega, \tau_{d}\right)=-\sum_{M}\left(\mu_{M}^{(\mathrm{pr})}\right)^{2} D_{M}^{\prime}(\omega) P_{M}\left(t_{\mathrm{pr}}\right)
$$

where $D_{M}^{\prime}(\omega)$ is the fluorescence lineshape function, Eq. (4.28). The $\operatorname{ESA}\left(\omega, \tau_{d}\right)$ is ${ }^{105}$

$$
\operatorname{ESA}\left(\omega, \tau_{d}\right)=\sum_{M, 2 N}\left(\mu_{M \rightarrow 2 N}^{(\mathrm{pr})}\right)^{2} D_{M \rightarrow 2 N}^{\prime}(\omega) P_{M}\left(t_{\mathrm{pr}}\right),
$$

with the lineshape function 


$$
\begin{aligned}
D_{M \rightarrow 2 N}^{\prime}(\omega)= & \operatorname{Re} \int_{0}^{\infty} d t e^{i\left(\omega-\widetilde{\omega}_{2 N M}^{\prime}\right) t} \\
& \times e^{G_{2 N M}(t)-G_{2 N M}(0)} e^{-t / \tau_{2 N M}},
\end{aligned}
$$

and

$$
G_{2 N M}(t)=\left(\gamma_{2 N 2 N}+\gamma_{M M}-2 \gamma_{2 N 2 N M M}\right) G(t) .
$$

The frequencies $\widetilde{\omega}_{2 N M}^{\prime}$ in Eq. (4.48) are

$$
\begin{aligned}
\widetilde{\omega}_{2 N M}^{\prime}= & \omega_{2 N M}^{\prime}-\frac{E_{\lambda}}{\hbar}\left(\gamma_{2 N 2 N}+\gamma_{M M}-2 \gamma_{2 N 2 N M M}\right) \\
& +\sum_{2 K \neq 2 N} \gamma_{2 N 2 K} \widetilde{C}^{(\operatorname{Im})}\left(\omega_{2 N 2 K}^{\prime}\right) \\
& +\sum_{K \neq M} \gamma_{K M}\left(\widetilde{C}^{(\operatorname{Im})}\left(\omega_{M K}^{\prime}\right)\right)^{*} .
\end{aligned}
$$

The shifted one-exciton energies $\omega_{M 0}^{\prime}$ are those in Eq. (4.25), and the two-exciton energies $\omega_{2 K 0}^{\prime}$ are

$$
\hbar \omega_{2 K 0}^{\prime}=\hbar \omega_{2 K 0}-2 E_{\lambda} \gamma_{2 K 2 K M M},
$$

where $E_{\lambda}$ is the reorganization energy in Eq. (4.17) and where

$\gamma_{2 K 2 K M M}=\sum_{m>n} \sum_{k}\left(c_{m n}^{(2 K)}\right)^{2}\left(c_{k}^{(M)}\right)^{2}\left(e^{-R_{m k} / R_{c}}+e^{-R_{n k} / R_{c}}\right)$,

and we have

$$
\omega_{\mu \nu}^{\prime}=\omega_{\mu 0}^{\prime}-\omega_{\nu 0}^{\prime} .
$$

Here $\mu$ and $\nu$ represent the different one- and two-exciton states in Eq. (4.51). The coupling constant $\gamma_{2 N 2 K}$ in Eq. (4.51) is

$$
\begin{aligned}
\gamma_{2 N 2 K}= & \sum_{m>n} \sum_{k>l}\left(c_{m n}^{(2 N)}\right)^{2}\left(c_{k l}^{(2 K)}\right)^{2}\left(e^{-R_{m k} / R_{c}}+e^{-R_{m l} / R_{c}}\right. \\
& +e^{\left.-R_{n k} / R_{c}+e^{-R_{n l} / R_{c}}\right) .}
\end{aligned}
$$

The inverse dephasing time in Eq. (4.49), $\tau_{2 N M}^{-1}=\tau_{2 N}^{-1}$ $+\left(\tau_{M}^{\prime}\right)^{-1}$, contains the one-exciton dephasing constant in Eq. (4.30) and the two-exciton constant $\tau_{2 N}^{-1}$ :

$$
\tau_{2 N}^{-1}=\sum_{2 K} \gamma_{2 N 2 K} \widetilde{C}^{(\mathrm{Re})}\left(\omega_{2 N 2 K}^{\prime}\right),
$$

where $\gamma_{2 N 2 K}$ is given by Eq. (4.55) and $\omega_{2 N 2 K}^{\prime}$ are obtained from Eqs. (4.52) and (4.54).

For large delay times $\tau_{d}=t_{\mathrm{pr}}-t_{\mathrm{pu}}$ the pump-probe signal becomes independent of $\tau_{d}$ because an equilibrium distribution of the exciton state occupation probabilities $P_{M}^{(\mathrm{eq})}$ is reached. The ground state bleaching $\mathrm{GB}(\omega)$ in Eq. (4.45) contains the effect of depopulation of the electronic ground state by the pump pulse and therefore is constant at times after the pump pulse. The stimulated emission $\operatorname{SE}\left(\omega, \tau_{d}\right)$ in Eq. (4.47) and excited state absorption $\operatorname{ESA}\left(\omega, \tau_{d}\right)$ in Eq. (4.48) contain information about the time-dependent population of exciton states: Only those one-exciton states which are populated at a given delay time $\tau_{d}$ will contribute to $\operatorname{SE}\left(\omega, \tau_{d}\right)$ and $\operatorname{ESA}\left(\omega, \tau_{d}\right)$.

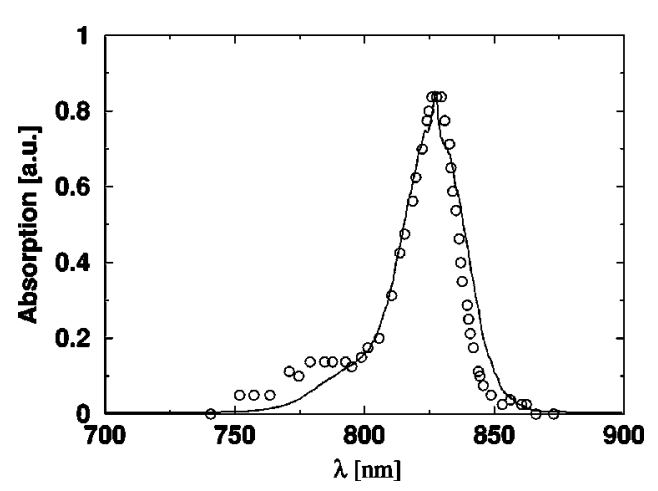

FIG. 5. Linear absorption spectrum of B820-complexes using the POP theory. Open circles are the experimental values from Ref. 2 and the line shows the calculation.

The equations in this section were used recently to calculate pump-probe spectra of Photosystem II reaction centers. ${ }^{45}$

\section{Fluorescence line narrowing spectra of B820 complexes}

The fluorescence line narrowing spectra ${ }^{2}$ of B820 complexes are calculated next using the spectral density $J(\omega)$ extracted from the fluorescence line narrowing spectra of the B777-complex in Sec. II. The B820 complex is modeled as a dimer of two B777 subunits with the intermolecular coupling of $230 \mathrm{~cm}^{-1}$ determined in Refs. $65-67$ from the circular dichroism and the difference spectra, singlet minus triplet. The geometry of optical transition dipoles was taken from the LH2 complex. ${ }^{3}$ The dependence of the fluorescence line narrowing spectra on the excitation energy $\hbar \omega_{\text {exc }}$ is given by the dimer analog of Eq. (2.1) for the monomer:

$$
I\left(\omega, \omega_{\mathrm{exc}}\right)=\left\langle\alpha\left(\omega_{\mathrm{exc}}\right) I(\omega)\right\rangle_{\text {disorder }},
$$

where $\alpha\left(\omega_{\text {exc }}\right)$ denotes the homogeneous absorption spectrum [Eqs. (4.1) and (4.18)] of the dimer complex and $I(\omega)$ is the homogeneous fluorescence spectrum given by Eqs. (4.27) and (4.28). In contrast to Eq. (2.1) for the monomer, where the disorder average was performed partly analytically, now the disorder average is performed numerically by a random generation of dimer site energies. A Gaussian distribution function of FWHM $=500 \mathrm{~cm}^{-1}$ and a mean site energy of the two pigments corresponding to $808 \mathrm{~nm}$ were determined from the fit of $1.6 \mathrm{~K}$ linear absorption spectrum, ${ }^{2}$ $\langle\alpha(\omega)\rangle_{\text {disorder }}$, using Eqs. (4.1) and (4.18). The POP theorycalculated and measured absorption spectra are compared in Fig. 5. For efficient numerical sampling of the disorder in Eq. (4.57), the $0 \rightarrow 0$ transition was broadened by adding an inverse dephasing time of $(2 \mathrm{ps})^{-1}$ to the inverse dephasing time $\tau_{M}^{-1}$ in Eq. (4.21). This additional broadening ${ }^{68}$ does not influence the shape of the vibrational sideband, because the latter has a large breadth from other sources. As noted before the $0 \rightarrow 0$ was not compared with the present experiment, because of scattered light from the excitation in the experiment. For the correlation radius of protein vibrations a value of $R_{c}=5 \AA$ was chosen, estimated in an earlier article ${ }^{45}$ from pump-probe spectra of PS-2 reaction centers. 


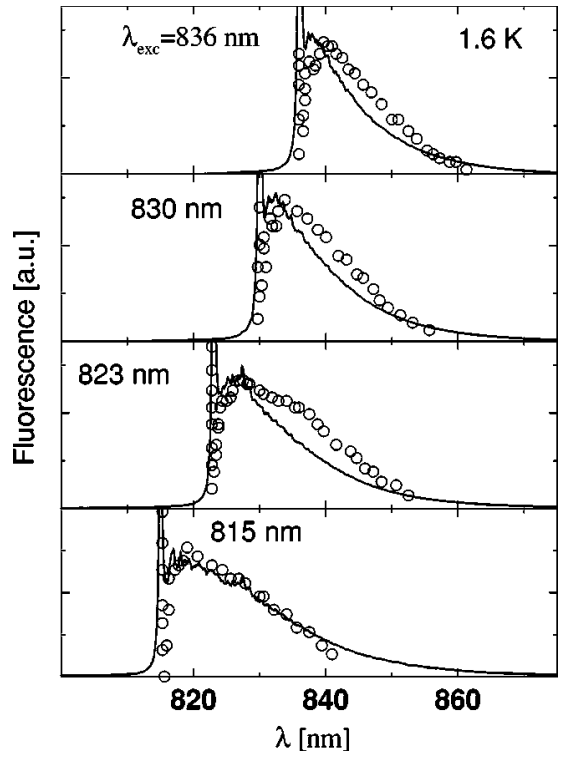

FIG. 6. Fluorescence line narrowing spectra of the B820-complexes for different excitation energies using the POP theory. Open circles are the experimental values from Ref. 2 and the lines show the calculations. The noise in the theoretical spectra is caused by the finite (10000) ensemble size of random configurations of disorder used in the calculation.

The calculation procedure consists of the following steps: (i) random generation of site energies, (ii) the calculation of the exciton energies and eigencoefficients by a diagonalization, (iii) the calculation of absorption $\alpha\left(\omega_{\text {exc }}\right)$ at the excitation energy, (iv) the calculation of the fluorescence spectrum $I(\omega)$, (v) performing the disorder average $\langle\cdots\rangle_{\text {disorder }}$ by repetition of steps (i)-(iv), and the summation of the results for $\alpha\left(\omega_{\text {exc }}\right) I(\omega)$. The fluorescence line narrowing spectra are calculated using this prescription and the parameters given above and are compared with the measured spectra $^{2}$ in Fig. 6.

An alternative partial summation of the excitonvibrational coupling was obtained by using a chronological ordering prescription (COP) in Appendix F. The integrodifferential equations for the density matrix obtained in a COP reveal a lineshape function similar to the Markovian Lorentzian lineshape given later in Eq. (5.4) but with a frequency-dependent broadening function that allows one to describe the high energy part of the vibrational side band, as seen in the calculation of fluorescence line narrowing spectra $^{69}$ in Fig. 8, which corresponds to Fig. 6 calculated with POP. The mean site energy of the pigments in the COP calculations was $806 \mathrm{~nm}$, determined from the linear absorption in Fig. 7.

\section{DISCUSSION}

\section{A. The correlation function $C(t)$}

The correlation function $C(t)$ of the energy gap of the pigment in the B777-complex is shown in Fig. 3. Although fluorescence line narrowing and hole burning spectra have been calculated for different pigment-protein complexes before ${ }^{50-53}$ we are not aware of a correlation function $C(t)$ having being extracted from such data. A second and merely technical difference from earlier work is the present use of

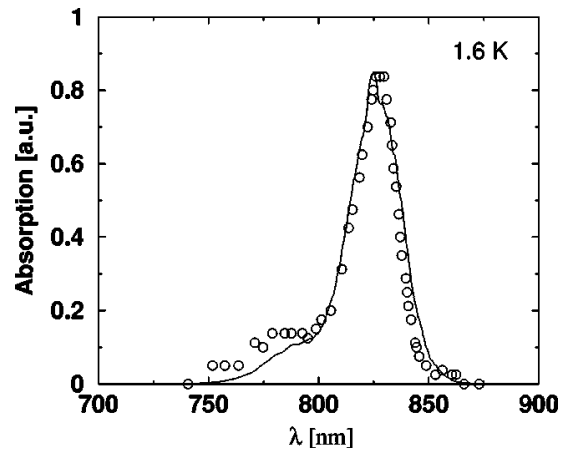

FIG. 7. Linear absorption spectrum of B820-complexes using a COP theory. Open circles are the experimental values from Ref. 2 and the line shows the calculation.

the FFT for the calculation of the spectra, which facilitates the use of a more general shape of the spectral density $J(\omega)$. Sometimes a combination of Gaussian and Lorentzian functional forms ${ }^{50}$ and sometimes ${ }^{51}$ a spectral density with $q$ $=2$ and $p=1$ in Eq. (2.16) are used instead, with $q=2$ being inferred from the Debye density of states for bulk crystals.

The shape of the $C(t)$ versus $t$ plot is needed in the subsequent theory. The major part of $C(t)$ in Fig. 3 is seen to decay on a $100 \mathrm{fs}$ time scale, and a small part (5\%) on a 500 fs time scale. One can infer that for any multi-pigment system composed of pigment-protein subunits of structure similar to that of the B777-complex, significant non-Markovian effects in the exciton transfer dynamics can be expected only when exciton transfer occurs on a 100 fs (or shorter) time scale. Such ultrafast transfer times have been observed in a number of antennas, among them the LH $2,{ }^{70} \mathrm{LH} 1,{ }^{70} \mathrm{LHCII}^{71}$ and the photosynthetic reaction centers of bacteria and green plants. ${ }^{72}$ In that case a non-Markovian theory is expected to provide a more detailed understanding of such experiments. As discussed in detail below an important feature that can be deduced from $C(t)$ concerns the appearance of vibrational sidebands in optical spectra, which has a non-Markovian origin.

\section{B. Fluorescence line narrowing spectra of B820-complexes}

The extracted $J(\omega)$ in Fig. 3 was used for the calculation of fluorescence line narrowing spectra of dimeric B820complexes. The resulting comparison with the experiment ${ }^{2}$ on B820-complexes is shown in Fig. 6. The non-Markovian theory predicts the correct shape of the vibrational sideband at different excitation energies. A small difference in the two inhomogeneous widths $600 \mathrm{~cm}^{-1}$ and $500 \mathrm{~cm}^{-1}$ deduced for the pigment transition energies of the B777-complex and the B820-complex might, if real, be due to a greater exposure of the B777 pigment to the solvent, ${ }^{73}$ as suggested in Ref. 2. The widths (FWHM) of $500 \mathrm{~cm}^{-1}$ and $600 \mathrm{~cm}^{-1}$ were determined here from the calculation of linear absorption spectra shown in Figs. 4 and 5. The additional narrowing of the main peak in the B820 absorption spectrum from $500 \mathrm{~cm}^{-1}$ for the pigments to $350 \mathrm{~cm}^{-1}$ for the excitonic transition in Fig. 5 is caused by the intermolecular coupling, the socalled resonance energy transfer narrowing. ${ }^{75}$ The inhomoge- 
neous broadening of exciton states in larger antenna complexes is smaller $\left(70-240 \mathrm{~cm}^{-1}\right)^{76-79}$ than the $350 \mathrm{~cm}^{-1}$ for B820, presumably because of lesser exposure to the solvent and stronger resonance energy transfer narrowing. The highenergy shoulder in the spectrum in Fig. 5 in the experimental and calculated spectrum is due to transition to the upper exciton state, which exhibits only small oscillator strength because of an unfavorable geometry of optical transition dipoles of the pigments. The fact that the experimental shoulder is more pronounced than the calculated one may arise from the additional unbound chlorophyll $a$ in the sample, an effect not taken into account here.

\section{The fast modulation limit: Markovian lineshapes}

The Markovian limit for the absorption and fluorescence lineshapes will be considered first. For this purpose the $\rho_{M 0}(t)$ in Eq. (4.12) is investigated and written as

$$
\rho_{M 0}(t)=e^{-i \Omega_{M} t} e^{-t / \tau_{M}} e^{-\gamma_{M M} \int_{0}^{t} d \tau(t-\tau) C(\tau)},
$$

where Eqs. (4.13) and (4.21) were used, and $\Omega_{M}=\omega_{M 0}$ $+\Sigma_{K \neq M} \gamma_{M K} \widetilde{C}^{\operatorname{Im}}\left(\omega_{M K}\right)$. The introduction of the dephasing time $\tau_{M}$ in Eq. (4.21) is based on a Markov approximation for the off-diagonal terms of the exciton-vibrational coupling and is valid if the exciton relaxation is slow when compared to the decay time $\tau_{c}$ of the exciton-vibrational correlation function $C(t)$, i.e., $\tau_{M}>\tau_{c}$. For the $C(t)$ in Fig. $3 \tau_{c} \approx 100 \mathrm{fs}$, neglecting the small amplitude tail of $C(t)$ that decays in a $500 \mathrm{fs}$ time scale. The Markovian limit for the absorption lineshape function is obtained by approximating

$$
e^{-\gamma_{M M} \int_{0}^{t} d \tau(t-\tau) C(\tau)} \approx e^{-\gamma_{M M} t \int_{0}^{\infty} d \tau C(\tau)} .
$$

This approximation is valid, if

$$
\gamma_{M M} C(0) \tau_{c}^{2} \ll 1,
$$

as discussed for a two-level system in Ref. 43. In this socalled fast modulation limit ${ }^{44}$ there is no time for the excitation of vibrational quanta and a Lorentzian lineshape function, centered at $\widetilde{\omega}_{M 0}$ in Eq. (4.19) is obtained

$$
D_{M}(\omega)=\frac{\tau_{M}^{-1}}{\left(\omega-\widetilde{\omega}_{M 0}\right)^{2}+\tau_{M}^{-2}} .
$$

The equality $\int_{0}^{\infty} d \tau C(\tau)=-i t \widetilde{C}^{\operatorname{Im}}(\omega=0)=-i t E_{\lambda} / \hbar$ that follows from Eqs. (4.15) and (4.16) with $\omega=\omega_{M K}=0$ was used. Similarly the Markovian result for the fluorescence lineshape function $D_{M}^{\prime}(\omega)$ is obtained by replacing the $\tau_{M}$ and $\widetilde{\omega}_{M 0}$ in Eq. (5.4) by the $\tau_{M}^{\prime}$ in Eq. (4.30) and the $\widetilde{\omega}_{M 0}^{\prime}$ in Eq. (4.29), respectively. Taking into account also ${ }^{80}$ $\widetilde{C}^{(\operatorname{Im})}\left(\omega_{M K}^{\prime}\right) \approx \widetilde{C}^{(\operatorname{Im})}\left(\omega_{M K}\right)$ and Eq. (4.25), the fluorescence and absorption lineshape functions peak at the same energy $\hbar \widetilde{\omega}_{M 0} \approx \hbar \widetilde{\omega}_{M 0}^{\prime}$, i.e., no Stokes shift is obtained in Markovian approximation, as expected ${ }^{14}$ in the fast modulation limit.

For the present system from Fig. 3 we estimate $\hbar^{2} C(0) \approx 300 \mathrm{meV}^{2}$ and $\tau_{c} \approx 100$ fs so that $\gamma_{0} \tau_{c}^{2} \approx 7 \gamma_{M M}$. The factor $\gamma_{M M}$ depends on the delocalization of excitons and vibrations, $\gamma_{M M}=\sum_{m, n}\left(c_{m}^{(M)}\right)^{2}\left(c_{n}^{(M)}\right)^{2} e^{-R_{m n} / R_{c}}$. For delocalized vibrations, $R_{c} \gg R_{m n}, \gamma_{M M}=1$ results, whereas for localized vibrations, $R_{c} \ll R_{m n}, \gamma_{M M}=\Sigma_{m}\left(c_{m}^{(M)}\right)^{4}$ which may

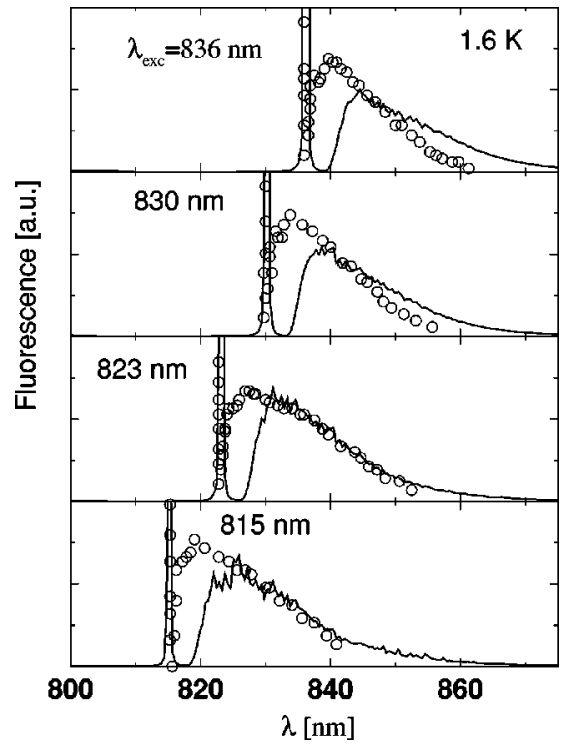

FIG. 8. Fluorescence line narrowing spectra of the B820-complexes for different excitation energies using a COP theory. Open circles are the experimental values from Ref. 2 and the lines show the calculations. The noise in the theoretical spectra is caused by the finite (10000) ensemble size of random configurations of disorder used in the calculation.

vary between 1 for localized electronic states and $1 / N, \mathrm{~N}$ being the number of pigments, for delocalized excitons. Hence, for the present dimer $1>\gamma_{M M}>1 / 2$ and Eq. (5.3) is not fulfilled. So, non-Markovian contributions to the lineshape functions are expected and they are discussed next.

\section{Non-Markovian lineshapes: Comparison of POP and COP}

A non-Markovian theory based on Eqs. (4.18) and (4.28) yields vibrational sidebands, in agreement with experiment. ${ }^{2}$ A partial ordering prescription (POP) gave the best agreement with the sideband obtained in the experiment as seen in the contrast between Figs. 6 and 8. The COP theory is seen to fail in the description of the low-energy part of the sideband, whereas the POP theory agrees reasonably with experiment in the whole spectrum, as seen in Fig. 6. In addition, the POP lineshape function, Eq. (4.18), reduces to the exact spin boson result, Eq. (2.7), in the limit of a twolevel system, i.e., for $\gamma_{M K}=\delta_{M K}$, whereas the COP theory does not. Hence, the nonperturbative summation ${ }^{81}$ of the diagonal part of the exciton vibrational coupling obtained in the POP theory but not in the COP led to a better agreement with the experiment.

The choice of ordering prescription used for a given system can be based on the statistical properties of the bath, as discussed by Mukamel in Ref. 43: If the dynamics of the bath can be mapped onto a random Gaussian process the partial ordering prescription is preferable, whereas a discrete stochastic process, for example, a two-state jump model is best included in a COP treatment of the lineshape function, ${ }^{43}$ as noted in the Introduction. The reaction coordinate for the present problem is the pigment's optical energy gap $X(t)$ that enters the correlation function $C(t)$ in Eq. (2.18). $X(t)$ is 
given as a linear combination of harmonic oscillator coordinates in Eq. (2.20). A combination of harmonic terms can be mapped onto a non-Markovian Gaussian stochastic process, irrespective of whether a quantum or classical description is used for the oscillators, shown by Mukamel ${ }^{82}$ using a generating function approach. Hence, the better agreement of POP for the present system is a consequence of the Gaussian properties of the reaction coordinate $X(t)$.

As seen in the B820 absorption spectra in Figs. 5 and 7 , the calculation of inhomogeneous spectra gives very similar results in both theories, POP and COP, due to the averaging. Due to the different sidebands in the two theories the mean site energies estimated from the calculation of the absorption spectra differ by $2 \mathrm{~nm}$, the COP site energy being lower in energy to compensate the more prominent contributions to the high-energetic part in the vibrational sideband. The numerical effort is somewhat less in the COP calculations.

\section{E. Excitonic potential energy surfaces}

We also used POP and COP theories recently to calculate various photophysical properties, among them time-resolved pump-probe spectra of PS-2 reaction centers. ${ }^{45}$ It was seen there that in the pump-probe spectra the exciton state relaxation did not change considerably when a Markov approximation was applied. The major effect of the latter was on the lineshape function of the optical transitions rather than on the time-dependent change of the spectrum. Since the exciton relaxation is determined by the off-diagonal terms of the exciton-vibrational coupling, whereas the vibrational sidebands are determined by the diagonal part of that coupling, it seems reasonable to apply, as we have done in the POP treatment, a Markov approximation to the off-diagonal terms and to describe the diagonal terms by a non-Markovian method. In doing so a first-order picture of mutually shifted excitonic PES arises, where the shift is given by the diagonal part of the exciton-vibrational coupling. The exciton relaxation occurs between such PES and is described by the off-diagonal part in Eq. (4.22). The diagonal part of each excitonic state is characterized by an excitonic reorganization energy $E_{\lambda}^{(M)}$ $=\gamma_{M M} E_{\lambda}$ and an excitonic Huang Rhys factor $S_{M}=\gamma_{M M} S$. Here, $E_{\lambda}$ is the reorganization energy of the local pigmentprotein coupling in Eq. (4.17) and $S$ is the local Huang Rhys factor. The value of $\gamma_{M M}$ depends on the delocalization of excitons and vibrations: Electronic delocalization leads to a decrease in the coupling $\gamma_{M M}$ to local protein vibrations.

The rate of exciton relaxation given in Eq. (4.22) contains the vibrational term $\widetilde{C}^{(\mathrm{Re})}\left(\omega_{M K}\right)$ in Eq. (4.15). This term describes how the protein can dissipate the electronic energy by absorption $\left(1+n\left(\omega_{M K}\right)\right)$ or emission $n\left(\omega_{K M}\right)$, of vibrational quanta, thus ensuring energy conservation during exciton relaxation. The electronic factor $\gamma_{M K}$ entering the rate in Eq. (4.22) is given in Eqs. (4.11) and (4.9). In the limit of delocalized protein vibrations $\left(R_{c} \gg R_{m n}\right) \quad \gamma_{M K}=|\langle M \mid K\rangle|^{2}$ $=\delta_{M K}$, and so no relaxation occurs, whereas for localized protein vibrations $\left(R_{c} \ll R_{m n}\right) \quad \gamma_{M K}=\Sigma_{m}\left(c_{m}^{(M)}\right)^{2}\left(c_{m}^{(K)}\right)^{2}$, which can be as small as zero for localized electronic states (e.g., if $c_{m}^{(M)}=1$ then $c_{m}^{(K)}=0$ for $K \neq M$ ). It equals $1 / N$ for completely delocalized excitons, $N$ being the number of pig- ments. Thereby, a fast exciton relaxation reflected in a large $\gamma_{M K}$ for $M \neq K$ is promoted by localized protein vibrations and partly delocalized electronic states. Delocalized protein vibrations could, however, lead to dissipation via the modulation of interpigment couplings, a modulation which was neglected here.

\section{F. Approximations}

Key approximations used in the present article are the following:

The secular approximation, used in the description of dissipation and described in Appendix B. The approximation has been investigated recently in a model study on a dimer ${ }^{83}$ and in a calculation of fluorescence depolarization in the B850 antenna complex of purple bacteria. ${ }^{84}$ The study of B850 complexes, which is the first nonsecular study of a photosynthetic antenna system, showed rather minor contributions from the nonsecular terms to the depolarization ${ }^{84}$ in the parameter range of most interest for experiment. ${ }^{85} \mathrm{~A}$ dimer model ${ }^{83}$ showed larger contributions which depended on the parameters. It would be useful to see whether the inclusion of nonsecular terms contributes more in line narrowing optical spectra, which can resolve the homogeneous lineshapes.

The third-order perturbation theory used in the present article for the coupling of excitons to the external fields restricts the theory to pump-probe spectra whose intensity is proportional to the intensity of the pump field. The experiments on pigment-protein complexes are usually performed in this regime, so simplifying the interpretation of the experiments. A notable exception is the investigation of excitonexciton annihilation in studies of the intensity dependence of pump-probe spectra (e.g., Ref. 86). The present neglect of the field-dependence of dissipation is well justified when the third-order perturbation theory is valid. ${ }^{87}$

In the calculation of pump-probe spectra a finite pump pulse and a delta function probe pulse were assumed. The pump pulse was chosen to be finite rather than a delta function in order to include a finite spectral width. That finite width is necessary to excite selectively certain exciton states, whereas a delta function probe pulse with its white spectrum is able to probe the whole spectrum. In the experiment such a white spectrum is often generated by a stochastic mixture of finite pulses with different carrier frequencies (e.g., Ref. 88). In this case the theory would contain the appropriate spectral properties, and the time-resolution would be different only for short delay times where the actual two pulses might overlap. In principle it is, however, possible to use also in the experiment a longer pump pulse for selective excitation and a shorter probe pulse for better time-resolution and a greater spectral width of detection.

In the calculation of the pump-probe spectra it was assumed that there was fast vibrational relaxation within an excitonic state and so coherences created by the pump pulse between different exciton states were neglected. This approximation was used to obtain a simple relation between time-dependent populations of exciton states, nonMarkovian optical lineshapes and pump-probe spectra. An inclusion of coherent vibrational motion found in some 
pigment-protein complexes ${ }^{89-94}$ is beyond the scope of the present approach, which considers equilibrium fluctuations of the vibrations. A Markov approximation could have been used to include coherences between different exciton states. $^{22}$

A correlation radius ${ }^{22}$ for the protein vibrations relates the correlation function of the exciton-vibrational coupling in the quantum master equation to a local correlation function $C(t)$. This $C(t)$ thus characterizes the modulation of the local pigment energies by the protein dynamics and is related to the local spectral density $J(\omega)$ in Eq. (2.22). $J(\omega)$ appears in the time-dependent formulation of the lineshape function in Eqs. (2.7) and (2.8), of the optical transition of the pigment and can thus be determined from independent experiments. A more detailed analysis of the molecular dynamics in photosynthetic proteins, using normal mode calculations or molecular dynamics simulations, could provide information on the size of the correlation radius $R_{c}$ and also on the importance of static and dynamic disorder in interpigment couplings, ${ }^{95}$ which was neglected here. It remains to be established whether the decay of the correlation function of the protein vibrations is indeed exponential, ${ }^{96}$ as assumed in Eq. (4.7).

\section{G. Comparison with other theories}

The present theory is related in spirit to a theory developed by Mukamel and co-workers ${ }^{23,24,97}$ and to a recent approach by Ohta, Yang and Fleming. ${ }^{98}$ The overall goal of these theories, including the present one, is to find an appropriate description of both the exciton-vibrational and the excitonic coupling. This problem also has a long history in the field of molecular crystals, where Green's function techniques were used to obtain results for linear optical spectra, as, for example in Refs. 99 and 100. A difference between molecular crystals and photosynthetic antennas is that in the latter the vibrational and electronic structure does not have the periodic symmetry found in the former. Even where the $\mathrm{X}$-ray structures appear symmetric as for the LH2 complex, ${ }^{3}$ disorder effects reduce the symmetry of the electronic and vibrational states. ${ }^{101}$ However, the general problem of finding an appropriate basis set of eigenfunctions remains. In the present paper the exciton states were defined for the equilibrium position of nuclei in the electronic ground state and the exciton-vibrational coupling was expressed in this basis as usual.

In the Liouville pathway correlation function approach employed by Mukamel and coworkers ${ }^{23}$ the diagonal part of the exciton-vibrational coupling is taken into account exactly, while the contributions to optical lineshape functions from the off-diagonal parts of the exciton-vibrational coupling were neglected. ${ }^{23}$ The present result for the linear absorption in Eqs. (4.1) and (4.18) reduces ${ }^{102}$ to their Eq. (3.3) in Ref. 23 by omitting all $\gamma_{M K}$ terms with $K \neq M$ in Eq. (4.19) and the exciton relaxation in (4.22). Ohta et al. ${ }^{98}$ recently combined Mukamel's correlation function approach with a standard Redfield approach, by introducing a lifetime broadening of the optical lineshapes. The present result of linear absorption in Eqs. (4.1) and (4.18) reduces to the one given in Eq. (16) in Ref. 98 when the exciton relaxationinduced shift of electronic energies, the terms with $\gamma_{M K}$ with $K \neq M$ in Eq. (4.19) are neglected.

In the presence of static disorder it can be expected that $\left|\gamma_{M K}\right|<\gamma_{M M}$ for $K \neq M$ and a large part of the shift of electronic energies is contained in the term with $\gamma_{M M}$ in Eq. (4.19). In this case the present POP theory of linear absorption and the theory in Ref. 98 can be expected to give similar results. One conceptional advantage of the present theory is that it rests solely on a density matrix formulation and is therefore more readily extended than another approach ${ }^{98}$ which combines two different theories, the density matrix theory and the correlation function approach. Extensions of the present theory are given in Appendices $\mathrm{C}$ and $\mathrm{F}$ by a non-Markovian treatment of the off-diagonal parts of the exciton-vibrational coupling in the POP theory (Appendix C) and in the COP theory (Appendix F).

Whereas in the calculation of the linear optical spectra the present theory contains the results of earlier correlation function approaches ${ }^{23,98}$ as limiting cases, in the case of nonlinear spectra there are nevertheless advantages of the correlation function approach. ${ }^{23}$ The main advantage of the latter is the inclusion of wave packet dynamics inside the excitonic PES which the present theory cannot describe. The theory of pump-probe spectra in Ref. 23 also contains coherent shorttime contributions which were neglected here. An advantage of the present theory is its relative simplicity.

The population relaxation rates obtained in the correlation function approach ${ }^{23,24,103}$ contain the mutual shifts of excitonic PES, whereas the present theory treats them in a standard Redfield type ${ }^{30}$ way. A recent comparison ${ }^{103}$ of both types of rates shows that they agree if the spectral density $J(\omega)$ is sufficient broad in energy to cover all transition energies between the different exciton levels. A Markovian approximation for the off-diagonal elements of the excitonvibrational coupling was applied in the present POP treatment as well as in the correlation function approach ${ }^{23}$ for the description of exciton relaxation. The COP theory in Appendix F uses a Fourier-Laplace transform to treat the equations of motion for the exciton state populations in a non-Markovian way. Non-Markovian contributions become important when the population relaxation is faster or on the same time scale as the vibrational relaxation and lead to nonexponential relaxation dynamics of excitons as discussed earlier.

\section{SUMMARY}

The theory developed in the present article includes nonMarkovian effects in the exciton-vibrational coupling in the calculation of frequency- and time-domain optical spectra of pigment-protein complexes. The presence of nonMarkovian effects was inferred from the presence of vibrational sidebands found at low temperatures in different pigment-protein complexes, since they are absent in a Markovian treatment. Additional evidence arises from a comparison of the decay of the correlation function of the optically probed energy gap of the pigments and ultrafast exciton re- 
laxation times measured in different antennas. ${ }^{70-72}$ The correlation function itself was estimated from fluorescence line narrowing spectra of B777-complexes.

The present theory provides a step towards a more microscopic understanding of the dynamic role of proteins in inducing exciton relaxation. This role appears in the rate constant $k_{M \rightarrow K}$ in Eq. (4.22) and its dependence on the delocalization of protein vibrations and excitons appears via $\gamma_{M K}$ and the spectral density $J(\omega)$ of the protein. The $J(\omega)$ was obtained from fluorescence line narrowing spectra of B777complexes. This $J(\omega)$ was used to calculate fluorescence line narrowing spectra of a two-pigment system, the B820 complex, using two different summations techniques for the exciton-vibrational coupling, POP and COP. Only one of the two, the second-order POP allows for an exact summation of the diagonal part of the exciton-vibrational coupling and therefore reduces to the exact result in the one-pigment limit. Only it gave agreement with the vibrational sideband found experimentally. This result was explained by the stochastic properties of the reaction coordinate $X(t)$, i.e., the optical energy gap of the pigments.

An interesting feature of the exciton-vibrational dynamics in photosynthetic antenna systems is the intermediate coupling strength of both the exciton-vibrational $\left(E_{\lambda}\right.$ $=100 \mathrm{~cm}^{-1}$, local reorganization energy estimated from the $J(\omega)$ of B777-complexes) and the excitonic coupling ( $V_{12}$ $=230 \mathrm{~cm}^{-1}$ in $\mathrm{B} 820$-complexes), a situation treated in the present approach by introducing excitonic potential energy surfaces.

As seen in our recent contribution ${ }^{45}$ on PS- 2 reaction centers, the theory is readily implemented numerically, including an average over disorder in electronic pigment energies and orientations of the different complexes in the sample.

\section{ACKNOWLEDGMENTS}

It is a pleasure to acknowledge support of a Lynen Research Fellowship from the Alexander von Humboldt Foundation to one of us (T.R.). The support of the National Science Foundation and the Office of Naval Research is also appreciated. We thank Dr. habil. V. May for stimulating discussions and we thank Professor S. Mukamel for many insightful comments.

\section{APPENDIX A: CORRELATION FUNCTION AND RELATION BETWEEN $\tilde{C}(\omega)$ AND SPECTRAL DENSITY $J(\omega)$}

The correlation function $C(t)$ in Eq. (2.22) is obtained, using Eqs. (2.20) and (2.21) and using $Q_{\xi}=C_{\xi}+C_{\xi}^{+}$. We have

$$
\begin{aligned}
C(t)= & \frac{1}{\hbar^{2}} \sum_{\xi}\left(\hbar \omega_{\xi}\right)^{2} g_{\xi}^{2} \operatorname{Tr}_{\mathrm{vib}}\left\{W _ { \mathrm { eq } } e ^ { i / \hbar H _ { \mathrm { vib } } t } \left(C_{\xi}\right.\right. \\
& \left.\left.+C_{\xi}^{+}\right) e^{-i / \hbar H_{\mathrm{vib}} t}\left(C_{\xi}+C_{\xi}^{+}\right)\right\},
\end{aligned}
$$

where $W_{\text {eq }}$ is the equilibrium statistical operator in Eq. (2.19). The vibrational Hamiltonian in Eq. (2.3) can be expressed in terms of the creation and annihilation operators as
$H_{\text {vib }}=\sum_{\xi} \hbar \omega_{\xi}\left(C_{\xi}^{+} C_{\xi}+1 / 2\right)$. Using the relation ${ }^{104}$ $e^{i \omega_{\xi} t C_{\xi}^{+} C_{\xi}} C_{\xi} e^{-i \omega_{\xi} t C_{\xi}^{+}} C_{\xi}=e^{-i \omega_{\xi} t} C_{\xi}$, one obtains

$$
C(t)=\sum_{\xi} \omega_{\xi}^{2} g_{\xi}^{2}\left\{\left(1+n\left(\omega_{\xi}\right)\right) e^{-i \omega_{\xi} t}+n\left(\omega_{\xi}\right) e^{i \omega_{\xi} t}\right\},
$$

where $\quad n\left(\omega_{\xi}\right)=\operatorname{Tr}_{\text {vib }}\left\{W_{\mathrm{eq}} C_{\xi}^{+} C_{\xi}\right\}=\Sigma_{N} N e^{-N \hbar \omega_{\xi} / k T /}$ $\Sigma_{M} e^{-M \hbar \omega_{\xi} / k T}=1 /\left(e^{\hbar \omega_{\xi} /(k T)}-1\right)$.

The correlation function can be expressed in terms of the spectral density in Eq. (2.9), as shown in Eq. (2.22). Applying the Fourier-Laplace transform Eq. (F5) to that equation then gives

$$
\begin{aligned}
\widetilde{C}(\omega)= & \lim _{\epsilon \rightarrow 0} \int_{0}^{\infty} d t e^{(i \omega-\epsilon) t} C(t) \\
= & \lim _{\epsilon \rightarrow 0} \int_{-\infty}^{\infty} d \omega^{\prime}\left(\omega^{\prime}\right)^{2}\left(1+n\left(\omega^{\prime}\right)\right)\left(J\left(\omega^{\prime}\right)\right. \\
& \left.-J\left(-\omega^{\prime}\right)\right) \frac{\epsilon+i\left(\omega-\omega^{\prime}\right)}{\left(\omega-\omega^{\prime}\right)^{2}+\epsilon^{2}} .
\end{aligned}
$$

Noting that the last factor in Eq. (A3) equals $\pi \delta\left(\omega-\omega^{\prime}\right)$ $+i \wp$, where $\wp$ denotes the Principal Part one arrives at Eqs. (4.15) and (4.16).

\section{APPENDIX B: EXCITON REPRESENTATION OF THE POP EQUATIONS AND CORRELATION FUNCTIONS OF THE EXCITON-VIBRATIONAL COUPLING}

The different phenomena in Sec. IV are treated using the equations of this Appendix. In the representation of exciton states $\mu, \nu, \ldots$ [Eq. (3.1)], the equation of motion (3.9) becomes

$$
\begin{aligned}
\frac{\partial}{\partial t} \rho_{\mu \nu}= & -i \omega_{\mu \nu} \rho_{\mu \nu}(t)+\frac{1}{i \hbar} \sum_{\kappa}\left(F_{\mu \kappa}(t) \rho_{\kappa \nu}(t)\right. \\
& \left.-F_{\kappa \nu}(t) \rho_{\mu \kappa}(t)\right)-\sum_{\kappa \lambda}\left\{K_{\mu \kappa \kappa \lambda}(t) \rho_{\lambda \nu}(t)\right. \\
& +K_{\nu \kappa \kappa \lambda}^{*}(t) \rho_{\mu \lambda}(t)-\left(K_{\lambda \nu \mu \kappa}(t)\right. \\
& \left.\left.+K_{\kappa \mu \nu \lambda}^{*}(t)\right) \rho_{\kappa \lambda}(t)\right\},
\end{aligned}
$$

where $\hbar \omega_{\mu \nu}=\mathcal{E}_{\mu}-\mathcal{E}_{\nu}$ [cf. Eq. (3.1)], and $K_{\mu \nu \kappa \lambda}(t)$ denotes the time-dependent dissipative function

$$
K_{\mu \nu \kappa \lambda}(t)=\int_{0}^{t-t_{0}} e^{-i \omega_{\kappa \lambda} \tau} C_{\mu \nu \kappa \lambda}(\tau),
$$

and $C_{\mu \nu \kappa \lambda}(t)$ denotes the exciton-vibrational correlation function for one- and two-exciton states,

$$
C_{\mu \nu \kappa \lambda}(t)=\left\langle\hat{V}_{\mu \nu}(t) \hat{V}_{\kappa \lambda}(0)\right\rangle,
$$

$\hat{V}_{\mu \nu}(t)$ being a matrix element of Eq. (3.13). The property $C_{\mu \nu \kappa \lambda}(t)=C_{\lambda \kappa \nu \mu}^{*}(-t)$ was used. 
The correlation function, Eq. (B3), can be expressed in terms of site correlation functions and exciton eigencoefficients for the one-exciton states $(\mu=M, \nu=N, \kappa=K$ and $\lambda=L$ ) as shown in Eq. (4.4). The respective correlation function for the two-exciton states is

$$
\begin{aligned}
C_{2 M 2 N 2 K 2 L}(t)= & \sum_{m>n} \sum_{k>l} c_{m n}^{(2 M)} c_{m n}^{(2 N)} c_{k l}^{(2 K)} c_{k l}^{(2 L)}\left\langle\left(\delta X_{m}(t)\right.\right. \\
& \left.\left.+\delta X_{n}(t)\right)\left(\delta X_{k}(0)+\delta X_{l}(0)\right)\right\rangle,
\end{aligned}
$$

and for the one- and two-exciton states we have

$$
\begin{aligned}
C_{2 M 2 N K L}(t)= & \sum_{m>n} \sum_{k} c_{m n}^{(2 M)} c_{m n}^{(2 N)} c_{k}^{(K)} c_{k}^{(L)}\left\langle\left(\delta X_{m}(t)\right.\right. \\
& \left.\left.+\delta X_{n}(t)\right) \delta X_{k}(0)\right\rangle .
\end{aligned}
$$

As demonstrated in the text the above correlation functions $C_{\mu \nu \kappa \lambda}(t)$ can be factorized into an electronic part $\gamma_{\mu \nu \kappa \lambda}$ and a vibrational part $C(t)$ by introducing a correlation radius $R_{c}$ of protein vibrations in Eq. (4.7),

$$
C_{\mu \nu \kappa \lambda}(t)=\gamma_{\mu \nu \kappa \lambda} C(t),
$$

where the factor $\gamma_{\mu \nu \kappa \lambda}$ depends on the exciton eigencoefficients and the correlation radius. The one-exciton function $\gamma_{M N K L}$ is given in Eq. (4.9). The two-exciton function $\gamma_{2 M 2 N 2 K 2 L}$ is

$$
\begin{aligned}
\gamma_{2 M 2 N 2 K 2 L}= & \sum_{m>n} \sum_{k>l} c_{m n}^{(2 M)} c_{m n}^{(2 N)} c_{k l}^{(2 K)} c_{k l}^{(2 L)}\left(e^{-R_{m k} / R_{c}}\right. \\
& \left.+e^{-R_{m l} / R_{c}}+e^{-R_{n k} / R_{c}}+e^{-R_{n l} / R_{c}}\right),
\end{aligned}
$$

and the mixed one- and two-exciton function $\gamma_{2 M 2 N K L}$ is

$$
\begin{aligned}
\gamma_{2 M 2 N K L}= & \sum_{m>n} \sum_{k} c_{m n}^{(2 M)} c_{m n}^{(2 N)} c_{k}^{(K)} c_{k}^{(L)}\left(e^{-R_{m k} / R_{c}}\right. \\
& \left.+e^{-R_{n k} / R_{c}}\right) .
\end{aligned}
$$

The above functions are used in the calculation of pumpprobe spectra in Eqs. (4.55) and (4.53), taking into account Eq. (B12).

The dissipative part of the equation of motion (B1) can be further simplified by introducing a rotating wave approximation for the dissipative parts, also known in the literature as a secular approximation. ${ }^{15,16,62}$ Specifically, only those elements of the sum $\Sigma_{\kappa \lambda}$ contribute which contain the same free time evolution $e^{i \omega_{\mu \nu} t}$ as the density matrix $\rho_{\mu \nu}$ on the left side of this equation. ${ }^{15,16,62}$ All other contributions lead to oscillating terms (nonsecular terms) in the solution which average to zero on a coarse-grained time axis. This secular approximation serves to decouple the equations of motion for the diagonal elements $\rho_{\mu \mu}(t)$, which are the exciton state occupation numbers, from those for the off-diagonal ele- ments $\rho_{\mu \nu}(t), \mu \neq \nu$, which describe the phase relationship of the different exciton states.

The respective dissipative parts of the equations of motion for the diagonal parts become

$$
\begin{aligned}
\left.\frac{\partial}{\partial t} \rho_{\mu \mu}(t)\right|_{\text {diss }} & -2 \sum_{\kappa \neq \mu}\left\{\operatorname{Re}\left\{K_{\mu \kappa \kappa \mu}(t)\right\} \rho_{\mu \mu}(t)\right. \\
& \left.-\operatorname{Re}\left\{K_{\kappa \mu \mu \kappa}(t)\right\} \rho_{\kappa \kappa}(t)\right\},
\end{aligned}
$$

and those for the off-diagonal elements are

$$
\begin{aligned}
\left.\frac{\partial}{\partial t} \rho_{\mu \nu}(t)\right|_{\text {diss }}= & -\left(\sum_{\kappa}\left(K_{\mu \kappa \kappa \mu}(t)+K_{\nu \kappa \kappa \nu}^{*}(t)\right)\right. \\
& \left.-2 \operatorname{Re}\left\{K_{\mu \mu \nu \nu}(t)\right\}\right) \rho_{\mu \nu}(t),
\end{aligned}
$$

where

$$
K_{\mu \kappa \kappa \mu}(t)=\gamma_{\mu \kappa} \int_{0}^{t-t_{0}} d \tau e^{i \omega_{\mu \kappa} \tau} C(\tau),
$$

with

$$
\gamma_{\mu \kappa} \equiv \gamma_{\mu \kappa \kappa \mu}
$$

and

$$
K_{\mu \mu \nu \nu}(t)=\gamma_{\mu \mu \nu \nu} \int_{0}^{t-t_{0}} d \tau C(\tau) .
$$

In Sec. IV the solution for the first-order density matrix element $\rho_{M 0}^{(1)}(t)$ on the right hand side of Eq. (4.38) is obtained from Eqs. (B1) and (B2), using Eqs. (4.36) and (4.37), applying the secular approximation to the dissipative terms [Eq. (B10)] and the Markov approximation to the offdiagonal part of the exciton-vibrational coupling as before, and setting $t_{0}=0$ in Eq. (B2). This choice of $t_{0}$ implies that the pump-pulse acts at positive times close to zero. The $\rho_{M 0}^{(1)}(t)$ then becomes

$$
\begin{aligned}
\rho_{M 0}^{(1)}(t)= & \frac{i \mu_{M}^{(p u)}}{\hbar} \int_{0}^{t} d \tau e^{-i \widetilde{\omega}_{M 0}(t-\tau)} e^{-(t-\tau) / \tau_{M}} \\
& \times e^{G_{M}(t)-G_{M}(\tau)} E_{\Omega_{p u}}(\tau) e^{-i \Omega_{p u} \tau},
\end{aligned}
$$

which may be conveniently calculated: A Fourier-Laplace transform is introduced to treat the convolution in Eq. (B14) and the inverse is then taken and Eq. (4.39) is obtained.

\section{APPENDIX C: NON-MARKOVIAN SOLUTION FOR OFF-DIAGONAL TERMS OF EXCITON-VIBRATIONAL COUPLING}

A non-Markovian description of the off-diagonal exciton-vibrational coupling in the calculation of the lineshape function for linear absorption is readily obtained: The non-Markovian solution of the integral in Eq. (4.13) is 


$$
\begin{aligned}
\gamma_{M K} \int_{0}^{t} d \tau(t-\tau) C(\tau) e^{i \omega_{M K} \tau} \\
=\gamma_{M K} \int_{0}^{\infty} d \omega \omega^{2} J(\omega) \\
\quad \times\left(( 1 + n ( \omega ) ) \left\{\frac{\cos \left(\left(\omega_{M K}-\omega\right) t\right)-1}{\left(\omega_{M K}-\omega\right)^{2}}\right.\right. \\
\left.\quad+i \frac{\sin \left(\left(\omega_{M K}-\omega\right) t\right)-\left(\omega_{M K}-\omega\right) t}{\left(\omega_{M K}-\omega\right)^{2}}\right\}+n(\omega) \\
\quad \times\left\{\frac{\cos \left(\left(\omega_{K M}-\omega\right) t\right)-1}{\left(\omega_{K M}-\omega\right)^{2}}\right. \\
\left.\left.\quad-i \frac{\sin \left(\left(\omega_{K M}-\omega\right) t\right)-\left(\omega_{K M}-\omega\right) t}{\left(\omega_{K M}-\omega\right)^{2}}\right\}\right)
\end{aligned}
$$

The Markovian limit, Eq. (4.13), is reached for large times $t \gg 1 / \omega_{M K}$.

\section{APPENDIX D: VIBRATIONALLY EQUILIBRATED EXCITED STATES AND EXCITONIC POTENTIAL ENERGY SURFACES}

In the following the diagonal part of the excitonvibrational coupling Hamiltonian of the one-exciton states, Eq. (3.3), is used to define excitonic potential energy surfaces (PES). ${ }^{29,30}$ The vibrational Hamiltonian in Eq. (2.3) was defined with respect to the equilibrium position of nuclei for the electronic ground state. To obtain a PES $U_{M}$ for an exciton state $|M\rangle$, it is useful when the system is vibrationally relaxed in that state, to combine the vibrational Hamiltonian and the diagonal part of the one-exciton part of the Hamiltonian of the exciton vibrational coupling, Eq. (3.2), in the usual fashion to obtain

$$
U_{M}=U_{M}^{(0)}+\sum_{\xi} \frac{\hbar \omega_{\xi}}{4}\left(Q_{\xi}+2 g_{\xi}(M, M)\right)^{2} .
$$

The coupling constants $g_{\xi}(M, N)$ are

$$
g_{\xi}(M, N)=\sum_{m n} c_{m}^{(M)} c_{n}^{(N)} g_{\xi}^{(m)}
$$

and the PES minimum is

$$
U_{M}^{(0)}=\mathcal{E}_{M}-\sum_{\xi} \hbar \omega_{\xi} g_{\xi}(M, M)^{2} .
$$

The introduction of this PES is useful only when the diagonal part of the exciton-vibrational coupling is larger than the off-diagonal part, i.e.,

$$
g_{\xi}(M, M)>\left|g_{\xi}(M, N)\right| \quad(M \neq N) .
$$

When the excitonic states are completely localized one sees from Eq. (D2) that $g_{\xi}(M, N) / g_{\xi}(M, M)=0$ for $M \neq N$. Hence, condition (D4) is fulfilled if the excitonic states are partly localized, for example, by static disorder. In this case it can be assumed that the new equilibrium position of the vibrations for a vibrationally relaxed exciton state $|M\rangle$ occurs at

$$
Q_{\xi}=Q_{\xi}^{(M)}=-2 g_{\xi}(M, M),
$$

according to Eq. (D1). A purely electronic density matrix is not able to describe this reorganization of the nuclei, since it treats the vibrations as a passive system staying in thermal equilibrium. However, there are situations where a knowledge of the dynamics of this reorganization is not needed, for example, in the study of steady-state fluorescence from equilibrated excited states. Alternatively, the intra-exciton PES relaxation might be so fast that in a study of pumpprobe spectra it can be assumed that the probe pulse will find vibrationally equilibrated excited states. Since the equilibrium position is known from Eq. (D5), the aggregate Hamiltonian, Eqs. (3.1)-(3.2), can be transformed using a new coordinate $Q_{\xi}^{\prime}=Q_{\xi}+2 g_{\xi}^{(M, M)}$ which reflects the new equilibrium position of nuclei in the exciton state $M$. To use the density matrix theory it is convenient to order the Hamiltonian with respect to the dependence on the coordinate $Q_{\xi}^{\prime}$. The vibrational Hamiltonian consists of the quadratic terms

$$
H_{\mathrm{vib}}^{\prime}=T_{\text {nucl }}+\sum_{\xi} \frac{\hbar \omega_{\xi}}{4}\left(Q_{\xi}^{\prime}\right)^{2} .
$$

The linear terms define the exciton-vibrational coupling Hamiltonian,

$$
\begin{aligned}
H_{\mathrm{ex}-\mathrm{vib}}^{\prime}= & -\sum_{\xi} \hbar \omega_{\xi} Q_{\xi}^{\prime} g_{\xi}(M, M)|0\rangle\langle 0| \\
& +\sum_{K, N} \sum_{\xi} \hbar \omega_{\xi} Q_{\xi}^{\prime}\left\{g_{\xi}(K, N)\right. \\
& \left.-\delta_{K N} g_{\xi}(M, M)\right\}|K\rangle\langle N| \\
& +\sum_{2 K, 2 N} \sum_{\xi} \hbar \omega_{\xi} Q_{\xi}^{\prime}\left\{g_{\xi}(2 K, 2 N)\right. \\
& \left.-\delta_{2 K 2 N} g_{\xi}(M, M)\right\}|2 K\rangle\langle 2 N|,
\end{aligned}
$$

where the two-exciton coupling constant $g_{\xi}(2 K, 2 N)$ is

$$
g_{\xi}(2 K, 2 N)=\sum_{m>n} c_{m n}^{(2 K)} c_{m n}^{(2 N)}\left(g_{\xi}^{(m)}+g_{\xi}^{(n)}\right) .
$$

The coordinate-independent terms contribute to the excitonic Hamiltonian,

$$
H_{\mathrm{ex}}^{\prime}=\sum_{K} \hbar \omega_{K 0}^{\prime}|K\rangle\left\langle K\left|+\sum_{2 K} \hbar \omega_{2 K 0}^{\prime}\right| 2 K\right\rangle\langle 2 K|,
$$

with the shifted excitonic energies of the one-exciton states,

$$
\hbar \omega_{K 0}^{\prime}=\hbar \omega_{K 0}-2 \sum_{\xi} \hbar \omega_{\xi} g_{\xi}(K, K) g_{\xi}(M, M) ;
$$

Eq. (4.25) then is obtained from the above equation, using Eqs. (D2), (4.6), (4.7), and (4.26). The shifted two-exciton energies $\hbar \omega_{2 K 0}^{\prime}$ are

$$
\hbar \omega_{2 K 0}^{\prime}=\hbar \omega_{2 K 0}-2 \sum_{\xi} \hbar \omega_{\xi} g_{\xi}(2 K, 2 K) g_{\xi}(M, M)
$$

and Eq. (4.52) follows using Eqs. (D8), (B5), (B6), (4.6), (4.7), and (4.53). 
Terms which are off-diagonal with respect to the exciton states have been neglected in $H_{\mathrm{ex}}^{\prime}$, using Eq. (D4).

The electronic energies are shifted and new coupling functions result from the changed exciton-vibrational Hamiltonian $H_{\mathrm{ex}-\mathrm{vib}}^{\prime}$. The electronic ground state now also couples to the vibrations

$$
\gamma_{00}^{\prime}=\gamma_{M M} .
$$

However, we also have

$$
\gamma_{M M}^{\prime}=0 \quad \text { and } \quad \gamma_{M M \nu \nu}^{\prime}=0 \quad(\nu=0, N, 2 N),
$$

because the new equilibrium position of $H_{\text {vib }}$ is at the minimum of the $M$ th excitonic PES.

The new two-exciton coupling constant $\gamma_{2 N 2 N}^{\prime}$ can be similarly expressed as

$$
\gamma_{2 N 2 N}^{\prime}=\gamma_{2 N 2 N}+\gamma_{M M}-2 \gamma_{2 N 2 N M M},
$$

where $\gamma_{2 N 2 N}$ is given by Eq. (4.55) with $2 K=2 N, \gamma_{M M}$ is given by Eqs. (4.11) and (4.9) with $K=M$ and $\gamma_{2 N 2 N M M}$ is given by Eq. (4.53) with $2 K=2 N$. The shifted excitonic energies, Eqs. (D10) and (4.52), as well as the new coupling constants in Eqs. (D12)-(D14) are used to calculate the fluorescence spectra in Appendix E and to calculate the contributions from excited state absorption and stimulated emission to the pump-probe spectra in the limit of fast intraexciton relaxation, Eqs. (4.47) and (4.48).

To apply the quantum master equation, Eqs. (B1) and (B2), to the third-order density matrix elements terms $\rho_{M 0}^{(3)}(t, M)$ and $\rho_{2 N M}^{(3)}(t)$, needed in the calculation of the pump-probe spectra, a coordinate transformation of the Hamiltonian to the equilibrium position of the $M$ th PES is used. The $\rho_{M 0}^{(3)}(t, 0)$ arises from the second-order ground state population $\rho_{00}^{(2)}(t)$ and therefore no coordinate transformation is needed there. The equations of motion, Eqs. (4.41)(4.43), are then obtained from the quantum master equation, Eqs. (B1) and (B2). For this purpose the secular approximation, Eq. (B10), for the dissipative terms, and the Markov approximation for the off-diagonal part of the excitonvibrational coupling are again introduced, and one sets $t_{0}$ $=t_{\mathrm{pr}}$ in Eq. (B2). This choice of $t_{0}$ takes into account that before the delta function probe pulse acts at $t_{\mathrm{pr}}$ the statistical operator of excitons and vibrations factorizes due to fast vibrational relaxation.

\section{APPENDIX E: DERIVATION OF EQUATION FOR THE FLUORESCENCE SPECTRUM}

The fluorescence spectrum $I(\omega)$ is obtained from a Fourier-Laplace transform of the dipole-dipole correlation function $D(t)$,

$$
I(\omega) \propto \operatorname{Re} \int_{0}^{\infty} d \tau e^{i \omega \tau} D(\tau),
$$

where

$$
D(t)=\operatorname{Tr}\left\{\hat{\mathbf{d}}^{\dagger} \cdot \hat{\mathbf{d}}(\tau) W_{\mathrm{eq}}(e)\right\},
$$

$W_{\text {eq }}(e)$ is defined below, and the dipole operator,

$$
\hat{\mathbf{d}}^{\dagger}=\sum_{M} \vec{\mu}_{M}|M\rangle\langle 0|,
$$

contains all transitions between the one-exciton manifold $|M\rangle$ and the ground state $|0\rangle$. The time-dependence of the dipole operator is defined with respect to the aggregate Hamiltonian of the ground state and the 1-exciton manifold:

$$
\hat{\mathbf{d}}(t)=U_{0}^{\dagger}(t) \hat{\mathbf{d}} U_{e}(t),
$$

where $U_{0}(t)=e^{-(i / \hbar) H_{0} t}$, and $U_{e}(t)=e^{-(i / \hbar) H_{e} t}$. The Hamiltonian $H_{e}$ contains the vibrational Hamiltonian, Eq. (2.3), and the one-exciton contributions of Eqs. (3.1) and (3.2). The equilibrium statistical operator of the one-exciton manifold is written as

$$
W_{\mathrm{eq}}(e)=\sum_{M} p(M) W_{\mathrm{eq}}^{\mathrm{vib}}(M),
$$

where $p(M)$ denotes the Boltzmann distribution of exciton occupation probabilities and $W_{\mathrm{eq}}^{\mathrm{vib}}(M)$ describes vibrations which are equilibrated with respect to the minimum position of the $M$ th excitonic PES.

Equation (E2) can be written equivalently as

$$
D(t)=\operatorname{Tr}\left\{\hat{\mathbf{d}} U_{e}(t) W_{\mathrm{eq}}(e) \cdot \hat{\mathbf{d}}^{\dagger} U_{0}^{\dagger}(t)\right\},
$$

and the trace with respect to the electronic degrees of freedom can be performed yielding

$$
D(t)=\sum_{M} \vec{\mu}_{M} \vec{B}_{M 0}(t),
$$

where a transition amplitude $\vec{B}(t)$ was introduced:

$$
\begin{aligned}
\hat{\mathbf{B}}(t) & =\operatorname{tr}_{\mathrm{vib}}\left\{U_{e}(t) W_{\mathrm{eq}}(e) \hat{\mathbf{d}}^{\dagger} U_{0}^{\dagger}(t)\right\} \\
& =\sum_{M} p(M) \vec{\mu}_{M} \operatorname{tr}_{\mathrm{vib}}\left\{U_{e}(t) W_{\mathrm{eq}}^{\mathrm{vib}}(M)|M\rangle\langle 0| U_{0}^{\dagger}(t)\right\} .
\end{aligned}
$$

We have

$$
\hat{\mathbf{B}}(0)=\sum_{M} p(M) \vec{\mu}_{M}|M\rangle\langle 0| .
$$

In case of a two-level system an exact analytical solution for the propagation of the statistical operator $W_{\mathrm{eq}}^{\mathrm{vib}}(M)|M\rangle\langle 0|$ appearing in Eq. (E8) can be obtained. However, for the multi-level system considered here an approximate solution is used. The latter is obtained using the density matrix theory considered in this paper. In this case the transition amplitude $B_{M 0}(t)$ is

$$
\vec{B}_{M 0}(t)=p(M) \vec{\mu}_{M} \rho_{M 0}(t),
$$

and from Eq. (E9) it follows that

$$
\rho_{M 0}(0)=1 \text {. }
$$

The fluorescence signal then is obtained from the real part of the transform $\widetilde{\rho}_{M 0}(\omega)$ as

$$
I(\omega) \propto \sum_{M} p(M)\left|\mu_{M}\right|^{2} \operatorname{Re} \widetilde{\rho}_{M 0}(\omega) .
$$


Using the concept of excitonic PES introduced in Appendix $\mathrm{D}$ to take into account vibrationally relaxed excitonic states, the equation of motion for the density matrix $\rho_{M 0}(t)$ reads, using a $\mathrm{POP}$ description, as

$$
\begin{aligned}
\frac{d}{d t} \rho_{M 0}(t)= & -i \omega_{M 0}^{\prime} \rho_{M 0}(t)-\int_{0}^{t} d \tau\left\{\sum_{K \neq M} \gamma_{M K} e^{i \omega_{M K}^{\prime} \tau}\right. \\
& \left.\times C(\tau)+\gamma_{M M} C^{*}(\tau)\right\} \rho_{M 0}(t),
\end{aligned}
$$

which yields the fluorescence signal in Eqs. (4.27) and (4.28).

Alternatively, a COP description for the dissipative parts gives

$$
\begin{aligned}
\frac{d}{d t} \rho_{M 0}(t)= & -i \omega_{M 0}^{\prime} \rho_{M 0}(t) \\
& -\int_{0}^{t} d \tau\left\{\sum_{K \neq M} \gamma_{M K} e^{-i \omega_{K 0}^{\prime} \tau} C(\tau)\right. \\
& \left.+\gamma_{M M} e^{-i \omega_{M 0}^{\prime} \tau} C^{*}(\tau)\right\} \rho_{M 0}(t-\tau) .
\end{aligned}
$$

The transform of the above equation is

$$
\begin{aligned}
& -i \omega \widetilde{\rho}_{M 0}(\omega)-\rho_{M 0}(t=0) \\
& \quad=-i \omega_{M 0}^{\prime} \widetilde{\rho}_{M 0}(\omega)-\Gamma_{M}^{\prime}(\omega) \widetilde{\rho}_{M 0}(\omega),
\end{aligned}
$$

with the $\Gamma_{M}^{\prime}(\omega)$ given later in Eq. (F9). By introducing this result for $\widetilde{\rho}_{M 0}(\omega)$ and Eq. (E11) into Eq. (E12), Eq. (F8) for the fluorescence lineshape is obtained, taking into account Eq. (4.27).

\section{APPENDIX F: TREATMENT OF PHENOMENA USING THE CHRONOLOGICAL ORDERING PRESCRIPTION (COP)}

The dissipative part of the equation of motion, Eq. (3.9), using a COP reads ${ }^{38}$ as

$$
\begin{aligned}
\left.\frac{\partial}{\partial t} \hat{\rho}(t)\right|_{\text {diss }}= & -\frac{1}{\hbar^{2}} \int_{0}^{t-t_{0}} d \tau \\
& \times\left\langle\mathcal{L}_{\text {ex-vib }}(t) e^{-i \mathcal{L}_{\text {ex }}(t-\tau)} \mathcal{L}_{\text {ex-vib }}(\tau)\right\rangle \rho(\tau),
\end{aligned}
$$

and in the exciton representation,

$$
\begin{aligned}
\left.\frac{\partial}{\partial t} \rho_{\mu \nu}\right|_{\text {diss }}= & -\sum_{\kappa \lambda} \int_{0}^{t-t_{0}} d \tau\left(e^{-i \omega_{\kappa \nu} \tau} C_{\mu \kappa \kappa \lambda}(\tau) \rho_{\lambda \nu}(t-\tau)\right. \\
& +e^{-i \omega_{\mu \kappa} \tau} C_{\nu \kappa \kappa \lambda}^{*}(\tau) \rho_{\mu \lambda}(t-\tau)-\left[e^{-i \omega_{\mu \lambda} \tau}\right. \\
& \left.\left.\times C_{\lambda \nu \mu \kappa}(\tau)+e^{-i \omega_{\kappa \nu} \tau} C_{\kappa \mu \nu \lambda}^{*}(\tau)\right] \rho_{\kappa \lambda}(t-\tau)\right),
\end{aligned}
$$

where $C_{\mu \nu \kappa \lambda}(t)$ denotes the exciton-vibrational correlation function, Eq. (B3). Applying a secular approximation as discussed in Appendix B the dissipative part for the diagonal elements $\rho_{\mu \mu}(t)$ becomes

$$
\begin{aligned}
\left.\frac{\partial}{\partial t} \rho_{\mu \mu}(t)\right|_{\text {diss }}= & 2 \sum_{\kappa} \gamma_{\mu \kappa} \operatorname{Re} \int_{0}^{t-t_{0}} d \tau\left\{e^{i \omega_{\kappa \mu} \tau} C(\tau) \rho_{\kappa \kappa}(t-\tau)\right. \\
& \left.-e^{i \omega_{\mu \kappa} \tau} C(\tau) \rho_{\mu \mu}(t-\tau)\right\} .
\end{aligned}
$$

The dissipative part for the off-diagonal elements is

$$
\begin{aligned}
\left.\frac{\partial}{\partial t} \rho_{\mu \nu}\right|_{\text {diss }} ^{\mu \neq \nu}= & -\int_{0}^{t-t_{0}} d \tau\left\{\Sigma _ { \kappa } \left(e^{-i \omega_{\kappa \nu} \tau} \gamma_{\mu \kappa} C(\tau)\right.\right. \\
& \left.+e^{-i \omega_{\mu \kappa} \tau} \gamma_{\nu \kappa} C^{*}(\tau)\right) \\
& \left.-2 \gamma_{\nu \nu \mu \mu} \operatorname{Re}\{C(\tau)\}\right\} \rho_{\mu \nu}(t-\tau) .
\end{aligned}
$$

The coupling constants $\gamma_{\mu \kappa}$ and $\gamma_{\nu \nu \mu \mu}$ in Eqs. (B12) and (B13) were used. To solve the integro-differential equations for the density matrix the Fourier-Laplace transform,

$$
\begin{aligned}
& \widetilde{F}(\omega)=\lim _{\epsilon \rightarrow 0} \int_{0}^{\infty} d t e^{(i \omega-\epsilon) t} F(t), \\
& F(t)=\frac{1}{2 \pi} \int_{-\infty}^{\infty} d \omega e^{-i \omega t} \widetilde{F}(\omega),
\end{aligned}
$$

is used. In an alternative approach auxiliary functions ${ }^{25}$ have been introduced to transform the set of integro-differential equations for the density matrix into a set of ordinary differential equations. The coupling to the external light field in Ref. 25 was included nonperturbatively, and a Lorentzian form of the spectral density was assumed. The present approach includes the external pump and probe fields in thirdorder perturbation theory and neglects the field dependence of dissipation. However, an arbitrary functional form of the spectral density is treated here. An alternative weak field perturbation approach, which is also not restricted to a special type of spectral density, employs an expansion in terms of Laguerre polynomials. ${ }^{26,27}$

\section{Calculation of linear absorption, circular dichroism and fluorescence}

The COP lineshape function for linear absorption and circular dichroism is ${ }^{28}$

$$
D_{M}(\omega, \mathrm{COP})=\frac{\Gamma_{M}^{(\mathrm{Re})}(\omega)}{\left(\omega-\omega_{M 0}-\Gamma_{M}^{(\mathrm{Im})}(\omega)\right)^{2}+\left(\Gamma_{M}^{(\mathrm{Re})}(\omega)\right)^{2}},
$$

where the $\Gamma_{M}(\omega)$ function is defined by

$$
\Gamma_{M}(\omega)=\sum_{K} \gamma_{M K} \widetilde{C}\left(\omega-\omega_{K 0}\right) .
$$

As shown in Appendix E, the lineshape function $D_{M}^{\prime}(\omega)$, entering the formula for fluorescence, Eq. (4.27), becomes in the COP description,

$$
D_{M}^{\prime}(\omega, \mathrm{COP})=\frac{\Gamma_{M}^{\prime(\mathrm{Re})}(\omega)}{\left(\omega-\omega_{M 0}^{\prime}-\Gamma_{M}^{\prime(\operatorname{Im})}(\omega)\right)^{2}+\left(\Gamma_{M}^{\prime(\mathrm{Re})}(\omega)\right)^{2}},
$$

with 


$$
\Gamma_{M}^{\prime}(\omega)=\sum_{K \neq M} \gamma_{M K} \widetilde{C}\left(\omega-\omega_{K 0}^{\prime}\right)+\gamma_{M M} \widetilde{C}^{*}\left(\omega_{M 0}^{\prime}-\omega\right)
$$

\section{Calculation of pump-probe spectra}

As in the POP case discussed in the text, a perturbation theory for the coupling to external fields is used and the equation of motion for the off-diagonal elements $\rho_{\mu \nu}^{(n)}(t)$ then is

$$
\begin{aligned}
\frac{\partial}{\partial t} \rho_{\mu \nu}^{(n)}(t) \stackrel{\mu \neq \nu}{=} & -i \omega_{\mu \nu} \rho_{\mu \nu}^{(n)}(t)-\int_{0}^{t} d \tau\left\{\Sigma _ { \kappa } \left(e^{-i \omega_{\kappa \nu} \tau} \gamma_{\mu \kappa} C(\tau)\right.\right. \\
& \left.\left.+e^{-i \omega_{\mu \kappa} \tau} \gamma_{\nu \kappa} C^{*}(\tau)\right)-2 \gamma_{\nu \nu \mu \mu} \operatorname{Re}\{C(\tau)\}\right\} \\
& \times \rho_{\mu \nu}^{(n)}(t-\tau)+\left.\frac{\partial}{\partial t} \rho_{\mu \nu}^{(n)}(t)\right|_{\mathrm{ex}-\mathrm{rad}},
\end{aligned}
$$

where the field part $\left.(\partial / \partial t) \rho_{\mu \nu}^{(n)}(t)\right|_{\text {ex-rad }}$ is given in Eq. (4.37). The Fourier-Laplace transform of the above equation is

$$
\begin{aligned}
-i \omega \widetilde{\rho}_{\mu \nu}^{(n)}(\omega)= & -i \omega_{\mu \nu} \widetilde{\rho}_{\mu \nu}^{(n)}(\omega)-\left\{\sum _ { \kappa } \left(\gamma_{\mu \kappa} \widetilde{C}\left(\omega-\omega_{\kappa \nu}\right)\right.\right. \\
& \left.+\gamma_{\nu \kappa}\left(\widetilde{C}\left(\omega_{\mu \kappa}-\omega\right)\right)^{*}\right) \\
& \left.-2 \gamma_{\mu \mu \nu \nu} \operatorname{Re}\{\widetilde{C}(0)\}\right\} \widetilde{\rho}_{\mu \nu}^{(n)}(\omega) \\
& +\left.\int_{0}^{\infty} d \tau e^{i \omega \tau} \frac{\partial}{\partial t} \rho_{\mu \nu}^{(n)}(\tau)\right|_{\text {ex-rad }} .
\end{aligned}
$$

In addition to the above off-diagonal elements an equation for the second-order diagonal elements $P_{M}(t)=\rho_{M M}^{(2)}(t)$ has to be solved, which is

$$
\begin{aligned}
\frac{\partial}{\partial t} P_{M}(t)= & -2 \Sigma_{K} \gamma_{M K}\left(\int_{0}^{t} d \tau \operatorname{Re}\left\{e^{i \omega_{M K} \tau} C(\tau)\right\} P_{M}(t-\tau)\right. \\
& \left.-\operatorname{Re}\left\{e^{i \omega_{K M} \tau} C(\tau)\right\} P_{K}(t-\tau)\right)+\mathcal{S}_{M M}^{(2)}(t),
\end{aligned}
$$

with the source term

$$
\mathcal{S}_{M M}^{(2)}(t)=\frac{2}{\hbar} \mu_{M}^{(\mathrm{pu})} \operatorname{Im}\left(e^{i \Omega_{\mathrm{pu}}{ }^{t}} \rho_{M 0}^{(1)}(t)\right) .
$$

The $\rho_{M 0}^{(1)}(t)$ on the right is obtained as the inverse Fourier transform of $\widetilde{\rho}_{M 0}^{(1)}(\omega)$ which is obtained from Eq. (F11) as

$$
\hbar \widetilde{\rho}_{M 0}^{(1)}(\omega)=-\frac{\mu_{M}^{(\mathrm{pu})} \widetilde{E}_{\Omega_{\mathrm{pu}}}\left(\omega-\Omega_{\mathrm{pu}}\right)}{\omega-\omega_{M 0}+i \Gamma_{M}(\omega)},
$$

where the $\Gamma_{M}$ function is given in Eq. (F7) and $\widetilde{E}_{\Omega_{\mathrm{pu}}}(\omega$ $\left.-\Omega_{\mathrm{pu}}\right)$ is the transform of the pump pulse envelope $E_{\Omega_{\mathrm{pu}}}(t)$.

To ensure existence of the integral in Eq. (F5), the $F(t)$ there should tend to zero as $t \rightarrow \infty$. With this goal ensured an equation of motion for

$$
\Delta P_{M}(t)=P_{M}(t)-P_{M}^{(\mathrm{eq})},
$$

is solved, where $P_{M}^{(\mathrm{eq})}$ is the asymptotic solution of Eq. (F12) for $t \rightarrow \infty$, and is obtained as

$$
P_{M}^{(\mathrm{eq})}=\rho_{M M}(t \rightarrow \infty)=\frac{e^{-\hbar \omega_{M 0} / k T}}{\sum_{N} e^{-\hbar \omega_{N 0} / k T}} \sum_{K} \widetilde{\mathcal{S}}_{K K}^{(2)}(0),
$$

where the detailed balance,

$$
\widetilde{C}^{(\mathrm{Re})}(\omega)=e^{-\hbar \omega / k T} \widetilde{C}^{(\mathrm{Re})}(-\omega),
$$

following from Eqs (4.15) was used.

In terms of a transform of Eq. (F15) and using Eqs. (F12) and (F16) the $\Delta \widetilde{P}_{M}(\omega)$ is given by

$$
\begin{aligned}
-i \omega \Delta & \widetilde{P}_{M}(\omega)+\Sigma_{N} \gamma_{M N}\left\{\left(\widetilde{C}\left(\omega_{M N}+\omega\right)\right.\right. \\
& \left.+\widetilde{C}^{*}\left(\omega_{M N}-\omega\right)\right) \Delta \widetilde{P}_{M}(\omega)-\left(\widetilde{C}\left(\omega_{N M}+\omega\right)\right. \\
& \left.\left.+\widetilde{C}^{*}\left(\omega_{N M}-\omega\right)\right) \Delta \widetilde{P}_{N}(\omega)\right\} \\
= & -P_{M}^{(\mathrm{eq})}+\frac{i}{\omega} P_{M}^{(\mathrm{eq})} \Sigma_{N} \gamma_{M N}\left(\left[\widetilde{C}\left(\omega_{M N}+\omega\right)\right.\right. \\
& \left.+\widetilde{C}^{*}\left(\omega_{M N}-\omega\right)\right]-e^{-\hbar \omega_{N M} / k T}\left[\widetilde{C}\left(\omega_{N M}+\omega\right)\right. \\
& \left.\left.+\widetilde{C}^{*}\left(\omega_{N M}-\omega\right)\right]\right)+\widetilde{\mathcal{S}}_{M M}^{(2)}(\omega),
\end{aligned}
$$

where $\widetilde{\mathcal{S}}_{M M}^{(2)}(\omega)$ is the Fourier-Laplace transform of the source term $\mathcal{S}_{M M}^{(2)}(t)$ in Eq. (F13). There is no singularity at $\omega=0$ in the second term on the rhs, since the subsequent bracket vanishes at $\omega=0$ because of the detailed balance, Eq. (F17).

As before, a delta function probe pulse at $t_{\mathrm{pr}}$ and fast intra-exciton PES relaxation are assumed in the calculation of the pump-probe signal, Eq. (4.35). The three contributions in Eq. (4.44) then are

$$
\mathrm{GB}(\omega)=-\sum_{M}\left(\mu_{M}^{(\mathrm{pr})}\right)^{2} D_{M}(\omega, \mathrm{COP}) \sum_{K} P_{K}\left(t_{\mathrm{pr}}\right),
$$

where $D_{M}(\omega, \mathrm{COP})$ is the COP absorption lineshape, Eq. (F6),

$$
\mathrm{SE}(\omega)=-\sum_{M}\left(\mu_{M}^{(\mathrm{pr})}\right)^{2} D_{M}^{\prime}(\omega, \mathrm{COP}) P_{M}\left(t_{\mathrm{pr}}\right),
$$

with the COP fluorescence lineshape $D_{M}^{\prime}(\omega, \mathrm{COP})$, Eq. (F8), and

$$
\operatorname{ESA}(\omega)=\sum_{M, 2 N}\left(\mu_{M \rightarrow 2 N}^{(\mathrm{pr})}\right)^{2} D_{M \rightarrow 2 N}(\omega, \mathrm{COP}) P_{M}\left(t_{\mathrm{pr}}\right),
$$

where the excited state absorption lineshape $D_{M \rightarrow 2 N}(\omega, \mathrm{COP})$ is

$$
D_{M \rightarrow 2 N}(\omega, \mathrm{COP})=-\operatorname{Im}\left(\frac{1}{\omega-\omega_{2 N M}^{\prime}+i \Gamma_{2 N}^{\prime}(\omega)}\right) .
$$

The damping function $\Gamma_{2 N}^{\prime}(\omega)$ is

$$
\begin{aligned}
\Gamma_{2 N}^{\prime}(\omega)= & \sum_{2 K}^{\prime} \gamma_{2 N 2 K} \widetilde{C}\left(\omega-\omega_{2 K M}^{\prime}\right)+\sum_{K}^{\prime} \gamma_{M K} \widetilde{C}\left(\omega_{2 N K}^{\prime}-\omega\right) \\
& +\gamma_{2 N 2 N}^{\prime} \widetilde{C}\left(\omega-\omega_{2 N M}^{\prime}\right),
\end{aligned}
$$


where the $\gamma_{2 N 2 K}$ and $\gamma_{2 N 2 N}^{\prime}$ are given in Eqs. (4.55), (D14). The shifted one-exciton energies $\omega_{M 0}^{\prime}$ are those in Eq. (4.25), the two-exciton energies $\omega_{2 N 0}^{\prime}$ are defined in Eq. (4.52) and $\omega_{2 N M}^{\prime}=\omega_{2 N 0}^{\prime}-\omega_{M 0}^{\prime}$.

${ }^{1}$ H. Noji, R. Yasuda, M. Yoshida, and K. J. Kinosita, Nature (London) $\mathbf{3 8 6}$, 299 (1997)

${ }^{2}$ T. M. H. Creemers, C. A. De Caro, R. W. Visschers, R. van Grondelle, and S. Völker, J. Phys. Chem. B 103, 9770 (1999).

${ }^{3}$ G. McDermott, S. M. Prince, A. A. Freer, A. M. HawthornthwaiteLawless, M. Z. Papiz, R. J. Cogdell, and N. W. Isaacs, Nature (London) 374, 517 (1995).

${ }^{4}$ S. Karrasch, P. A. Bullough, and R. Gosh, EMBO J. 14, 631 (1995).

${ }^{5}$ K.-H. Rhee, E. P. Morris, J. Barber, and W. Kühlbrandt, Nature (London) 396, 283 (1998).

${ }^{6}$ B. Hankamer, E. P. Morris, and J. Barber, Nat. Struct. Biol. 6, 560 (1999).

${ }^{7}$ R. Kubo and Y. Toyozawa, Prog. Theor. Phys. 13, 160 (1955).

${ }^{8}$ M. Lax, J. Chem. Phys. 20, 1752 (1952).

${ }^{9}$ R. A. Marcus, J. Chem. Phys. 24, 966 (1956).

${ }^{10}$ V. G. Levich and R. R. Dogonadze, Dokl. Akad. Nauk SSSR 124, 123 (1959).

${ }^{11}$ Y. Georgievskii, C.-P. Hsu, and R. A. Marcus, J. Chem. Phys. 110, 5307 (1999).

${ }^{12}$ R. F. Loring, Y. J. Yan, and S. Mukamel, J. Chem. Phys. 87, 5840 (1987).

${ }^{13}$ D. Chandler, in Liquids, Freezing, and Glass Transition, edited by J. P. Hansen, D. Levesque, and J. Zinn-Justin (Elsevier, Amsterdam, 1991), p. 193.

${ }^{14}$ S. Mukamel, Principles of Nonlinear Optical Spectroscopy (Oxford University Press, New York, 1995), pp. 34, 45-75, 219, 222.

${ }^{15}$ K. Blum, Density Matrix Theory and Application (Plenum, New York, 1981), pp. 261-299.

${ }^{16}$ V. May and O. Kühn, Charge and Energy Transfer Dynamics in Molecular Systems: A Theoretical Introduction (Wiley-VCH, Berlin, 2000), pp. 103-113, 137, 203, 233.

${ }^{17}$ O. Kühn and V. May, J. Chem. Phys. 101, 10404 (1994).

${ }^{18}$ R. Kubo, M. Toda, and N. Hashitsume, Statistical Physics II (Springer Series in Solid State Physics, Heidelberg, 1985), pp. 204-263.

${ }^{19}$ V. Chernyak, N. J. Wang, and S. Mukamel, Phys. Rep. 263, 213 (1995).

${ }^{20}$ J. A. Leegwater, J. R. Durrant, and D. R. Klug, J. Phys. Chem. B 101, 7205 (1997).

${ }^{21}$ O. Kühn and V. Sundström, J. Chem. Phys. 107, 4154 (1997).

${ }^{22}$ T. Renger and V. May, J. Phys. Chem. A 102, 4381 (1998).

${ }^{23}$ W. M. Zhang, T. Meier, V. Chernyak, and S. Mukamel, Philos. Trans. R. Soc. London, Ser. A 356, 405 (1998).

${ }^{24}$ W. M. Zhang, T. Meier, V. Chernyak, and S. Mukamel, J. Chem. Phys. 108, 7763 (1998).

${ }^{25}$ C. Meier and D. J. Tannor, J. Chem. Phys. 111, 3365 (1999).

${ }^{26}$ T. Mancal and V. May, J. Chem. Phys. 114, 1510 (2000).

${ }^{27}$ T. Mancal and V. May, Chem. Phys. 268, 201 (2001).

${ }^{28}$ T. Renger and V. May, Phys. Rev. Lett. 84, 5228 (2000).

${ }^{29}$ T. Renger, Ph.D. thesis, Humboldt-University Berlin 1998, http:// dochost.rz.hu-berlin.de/dissertationen/physik/renger-thomas/

${ }^{30}$ T. Renger, V. May, and O. Kühn, Phys. Rep. 343, 138 (2001).

${ }^{31}$ A. Matro and J. Cina, J. Phys. Chem. 99, 2568 (1995).

${ }^{32}$ T. Renger and V. May, Phys. Rev. Lett. 78, 3406 (1997).

${ }^{33}$ N. Makri, E. Sim, D. E. Makarov, and M. Topaler, Proc. Natl. Acad. Sci. U.S.A. 93, 3926 (1996)

${ }^{34}$ J. Ray and N. Makri, J. Phys. Chem. A 103, 9417 (1999).

${ }^{35}$ R. Kubo, J. Math. Phys. 4, 174 (1963).

${ }^{36}$ N. G. van Kampen, Physica (Amsterdam) 74, 215,239 (1974).

${ }^{37}$ N. Hashitsume, F. Shibata, and M. Shingu, J. Stat. Phys. 17, 155 (1977).

${ }^{38}$ S. Mukamel, I. Oppenheim, and J. Ross, Phys. Rev. A 17, 1988 (1978).

${ }^{39}$ S. Chaturvedi and F. Shibata, Z. Phys. B: Condens. Matter 35, 297 (1979).

${ }^{40}$ M. Tokuyama and H. Mori, Prog. Theor. Phys. 55, 411 (1976).

${ }^{41}$ R. Zwanzig, J. Chem. Phys. 33, 1338 (1960).

${ }^{42}$ M. A. Palenberg, R. Silbey, C. Warns, and P. Reineker, J. Chem. Phys. 114, 4386 (2001).

${ }^{43}$ S. Mukamel, Chem. Phys. 37, 33 (1979).

${ }^{44}$ R. Kubo, in Fluctuation, Relaxation and Resonance in Magnetic Systems, edited by D. ter Haar (Oliver \& Boyd, Edinburgh, 1962), pp. 23-68.

${ }^{45}$ T. Renger and R. A. Marcus, J. Phys. Chem. B 106, 1809 (2002).

${ }^{46}$ R. I. Personov, in Spectroscopy and Excitation Dynamics of Condensed Molecular Systems, edited by V. M. Agranovich and R. M. Hochstrasser
(Elsevier/North-Holland, Amsterdam, 1983), pp. 555-619.

${ }^{47}$ A. J. Leggett, S. Chakravarty, A. T. Dorsey, M. P. A. Fisher, A. Garg, and W. Zwerger, Rev. Mod. Phys. 59, 1 (1987)

${ }^{48}$ The $G(t)$ here corresponds to the $\Delta G(t)$ introduced in Ref. 16 on p. 206.

${ }^{49}$ The $n(\omega)$ is obtained from $\Sigma_{N} N e^{-N \hbar \omega} / \Sigma_{M} e^{-M \hbar \omega}$

${ }^{50}$ J. Pieper, J. Voigt, G. Renger, and G. J. Small, Chem. Phys. Lett. 310, 296 (1999).

${ }^{51}$ T. Pullerits, R. Monshouwer, F. van Mourik, and R. van Grondelle, Chem. Phys. 194, 395 (1995).

${ }^{52}$ E. J. G. Peterman, H. van Amerongen, R. van Grondelle, and J. P. Decker, Proc. Natl. Acad. Sci. U.S.A. 95, 6128 (1998).

${ }^{53}$ E. J. G. Peterman, T. Pullerits, R. van Grondelle, and H. van Amerongen, J. Phys. Chem. B 101, 4448 (1997).

${ }^{54}$ M. Rätsep, W. T. Johnson, P. R. Chitnis, and G. J. Small, J. Phys. Chem. B 104, 836 (2000).

${ }^{55}$ B. Gobets and R. van Grondelle, Biochim. Biophys. Acta 1507, 80 (2001).

${ }^{56}$ V. Zazubovich, S. Matsuzaki, T. W. Johnson, J. M. Hayes, P. R. Chitnis, and G. J. Small, Chem. Phys. 275, 47 (2002).

${ }^{57}$ In principle, the scattered light that interfers with the $0 \rightarrow 0$ transition in the spectrum could be eliminated by using pulsed instead of cw excitation. In this case the scattered light could be avoided by detecting the fluorescence signal after the scattered light of the excitation pulse has disappeared. However, the desired pulse width might be too small to achieve the spectral resolution necessary to resolve the $0 \rightarrow 0$ transition at $1.6 \mathrm{~K}$ [Ryszard Jankowiak (private communication)].

${ }^{58}$ This value is a compromise between the fits of fluorescence line narrowing and of linear absorption.

${ }^{59}$ Since the matrix containing the excitonic couplings and site energies is symmetric the eigenvalues obtained are real and real eigenvectors are calculated.

${ }^{60}$ An alternative and more general way is given by Mukamel's Liouville pathway correlation function approach (Refs. 23 and 24), which avoids a factorization of certain parts of the correlation between excitonic and vibrational degrees of freedom in the calculation of nonlinear optical spectra. The present theory can only treat these correlations exactly in the linear optical spectra and assumes a factorization of the statistical operator in the calculation of the nonlinear spectra.

${ }^{61}$ J. H. Freed, J. Chem. Phys. 49, 376 (1968).

${ }^{62}$ J. Breton, A. Hardisson, F. Mauricio, and S. Velasco, Phys. Rev. A 30, 553 (1984).

${ }^{63}$ Since $J(\omega)=0$ for $\omega<0$ the $J(-\omega) n(-\omega)$ only contributes for $\omega<0$ to the $\widetilde{C}^{(\mathrm{Re})}(\omega)$ in Eq. $(4.15)$.

${ }^{64}$ H. L. Friedman, A Course in Statistical Mechanics (Prentice-Hall, Englewood Cliffs, NJ, 1985), p. 299.

${ }^{65}$ R. W. Visschers, M. C. Chang, F. van Mourik, P. S. Parkes-Loach, B. A. Heller, P. A. Loach, and R. van Grondelle, Biochemistry 30, 5734 (1991).

${ }^{66}$ F. van Mourik, C. J. R. van der Oord, K. J. Visscher, P. S. Parkes-Loach, P. A. Loach, R. W. Visschers, and R. van Grondelle, Biochim. Biophys. Acta 1059, 111 (1991).

${ }^{67}$ M. H. C. Koolhaas, G. van der Zwan, F. van Mourik, and R. van Grondelle, Biophys. J. 72, 1828 (1997).

${ }^{68}$ The actual zero-zero transition exhibits, besides the population transfer induced broadening, an additional broadening due to pure net electronic dephasing effects that arise from quadratic coupling terms in the excitonvibrational coupling (for example, Ref. 30) that were neglected here. These quadratic terms describe the excitation/deexcitation of a vibrational quantum and lead to a "pure" electronic dephasing. However, the broadening by such dephasing is much weaker than the artificial broadening assumed here for the efficient numerical sampling of disorder.

${ }^{69}$ In the COP calculations it was necessary to correct the vibrationally equillibrated exciton energies $\hbar \omega_{M 0}^{\prime}$ in Eq. (D10) in order to ensure that the $0 \rightarrow 0$ transitions in absorption and fluorescence occur at the same energy. The energies $\hbar \omega_{M 0}^{\prime}$ were corrected by replacing the reorganization energy $E_{\lambda}$ in Eq. (D10) by a new energy $E^{\prime}=0.7 E_{\lambda}$. Such a correction was necessary in COP but not in POP because the treatment of the diagonal part of the exciton vibrational coupling in COP is perturbational and the treatment of this coupling in POP is exact. Therefore, in COP only part of the reorganization energy of intra-exciton PES relaxation is described. The shape of the vibrational sideband was not influenced by this correction.

${ }^{70}$ V. Sundström, T. Pullerits, and R. van Grondelle, J. Phys. Chem. B 103, 2346 (1999) 
${ }^{71}$ H. van Amerongen and R. van Grondelle, J. Phys. Chem. B 105, 604 (2001).

${ }^{72}$ J. R. Durrant, G. H. Hastings, D. M. Joseph, J. Barber, G. Porter, and D. R. Klug, Proc. Natl. Acad. Sci. U.S.A. 89, 11632 (1992).

${ }^{73}$ The inhomogeneous widths of the absorption lineshape measured for $\mathrm{BChl}_{a}$ in different polar solvents lie between $620 \mathrm{~cm}^{-1}$ and $930 \mathrm{~cm}^{-1}$ (Ref. 74).

${ }^{74}$ M. Becker, V. Nagarajan, and W. W. Parson, J. Am. Chem. Soc. 113, 6840 (1991).

${ }^{75}$ E. W. Knapp, Chem. Phys. 85, 73 (1984).

${ }^{76}$ S. G. Johnson and G. J. Small, J. Phys. Chem. 95, 471 (1991)

${ }^{77}$ J. Pieper, M. Rätsep, R. Jankowiak, K.-D. Irrgang, J. Voigt, G. Renger, and G. J. Small, J. Phys. Chem. A 103, 2412 (1999).

${ }^{78}$ N. R. S. Reddy, G. J. Small, M. Seibert, and R. Picorel, Chem. Phys. Lett. 181, 391 (1991).

${ }^{79}$ N. R. S. Reddy, R. J. Cogdell, L. Zhao, and G. J. Small, Photochem. Photobiol. 57, 35 (1993).

${ }^{80}$ From the coincidence of the sharp peaks with the excitation wavelengths in the POP fluorescence line narrowing spectra in Fig. 6 it can be concluded that the $0 \rightarrow 0$ transitions in absorption and fluorescence occur at the same energy, i.e., $\widetilde{\omega}_{M 0} \approx \widetilde{\omega}_{M 0}^{\prime}$, and from Eqs. (4.19) and (4.29) it then follows $\widetilde{C}^{(\mathrm{Im})}\left(\omega_{M K}^{\prime}\right) \approx \widetilde{C}^{(\mathrm{Im})}\left(\omega_{M K}\right)$.

${ }^{81}$ The Bloch-De Dominici theorem (Ref. 18), also known as Wick's theorem, leads to a factorization of higher order expectation values of the diagonal elements of the exciton-vibrational coupling, which is why the higher-order POP cumulants vanish exactly. The general structure of those was first derived by van Kampen (Ref. 36). Wick's theorem, however, does not apply to the off-diagonal elements occurring in a multi-pigment system in addition to the diagonal elements.

${ }^{82}$ S. Mukamel, J. Chem. Phys. 79, 2126 (1983)

${ }^{83}$ V. Capek, I. Barvik, and P. Herman, Chem. Phys. 270, 141 (2001).

${ }^{84}$ O. Kühn, V. Sundström, and T. Pullerits, Chem. Phys. 275, 15 (2002).

${ }^{85}$ See Fig. 8 in Ref. 84.

${ }^{86}$ T. Bittner, K. D. Irrgang, G. Renger, and M. R. Wasielewski, J. Phys. Chem. 98, 11821 (1994).

${ }^{87}$ D. H. Schirrmeister and V. May, Chem. Phys. 220, 1 (1997).

${ }^{88}$ H. M. Visser, M. L. Groot, F. van Mourik, I. H. M. van Stokkum, J. P. Dekker, and R. van Grondelle, J. Phys. Chem. 99, 15304 (1995).
${ }^{89}$ M. H. Vos, F. Rappaport, J. C. Lambry, J. Breton, and J. L. Martin, Nature (London) 363, 320 (1993); M. H. Vos, M. R. Jones, C. N. Hunter, J. Breton, and J. L. Martin, Proc. Natl. Acad. Sci. U.S.A. 91, 12701 (1994).

${ }^{90}$ M. Chachisvilis, H. Fidder, T. Pullerits, and V. Sundström, J. Raman Spectrosc. 26, 513 (1995).

${ }^{91}$ S. E. Bradforth, R. Jimenez, F. van Mourik, R. van Grondelle, and G. F. Fleming, J. Phys. Chem. B 99, 16179 (1995).

${ }^{92}$ R. Kumble, S. Palese, R. W. Visschers, P. L. Dutton, and R. B. Hochstrasser, Chem. Phys. Lett. 261, 396 (1996).

${ }^{93}$ W. M. Diffey, B. J. Homoelle, M. D. Edington, and W. F. Beck, J. Phys. Chem. B 102, 2776 (1998).

${ }^{94}$ R. Monshouwer, A. Baltuska, F. van Mourik, and R. van Grondelle, J. Phys. Chem. A 102, 4360 (1998).

${ }^{95}$ S. Jang, S. E. Dempster, and R. Silbey, J. Phys. Chem. B 105, 6655 (2001).

${ }^{96}$ More structural parameters than just the spatial distance between the two pigments may be important. The correlation radius is likely to be a function of the frequency of the normal modes, since high frequency modes can be expected to be more localized.

${ }^{97}$ A. Okada, V. Chernyak, and S. Mukamel, J. Phys. Chem. A 102, 1241 (1998).

${ }^{98}$ K. Ohta, M. Yang, and G. R. Fleming, J. Chem. Phys. 115, 7609 (2001).

${ }^{99}$ S. Fischer and S. A. Rice, J. Chem. Phys. 52, 2089 (1970).

${ }^{100}$ M. K. Grover and R. Silbey, J. Chem. Phys. 52, 2099 (1970).

${ }^{101}$ R. Monshouwer, M. Abrahamson, F. van Mourik, and R. van Grondelle, J. Phys. Chem. B 101, 7241 (1997).

${ }^{102}$ The functions $G(t)$ in Eqs. (2.8), (4.20) and (4.18) and the $g(t)$ used in Refs. 23 and 28 are related by (Ref. 14) $g(t)=G(0)-G(t)-i t E_{\lambda} / \hbar$.

${ }^{103}$ M. Yang and G. R. Fleming, Chem. Phys. 275, 355 (2002).

${ }^{104}$ H. Haken, Quantum Field Theory of Solids: An Introduction (NorthHolland, New York, 1976), p. 39.

${ }^{105}$ The equations for $\operatorname{GB}(\omega), \operatorname{SE}(\omega)$, and $\operatorname{ESA}(\omega)$ were printed incorrectly earlier in Ref. 45 in an application of the present theory. The present factor $\mu_{M}^{(\mathrm{pr})}$ in Eqs. (4.44), (4.45), (F19), and (F20) and the factor $\mu_{M \rightarrow 2 N}^{(\mathrm{pr})}$ in Eq. (4.48) are missing in Eqs. (21)-(23) in Ref. 45. The numerical calculations in Ref. 45 were performed with the correct equations given here. 